\title{
The Road Towards 6G: A Comprehensive Survey
}

\author{
Wei Jiang, Senior Member, IEEE, Bin Han, Member, IEEE, \\ Mohammad Asif Habibi, and Hans Dieter Schotten, Member, IEEE
}

As of today, the fifth generation (5G) mobile communication system has been rolled out in many countries and the number of 5G subscribers already reaches a very large scale. It is time for academia and industry to shift their attention towards the next generation. At this crossroad, an overview of the current state of the art and a vision of future communications are definitely of interest. This article thus aims to provide a comprehensive survey to draw a picture of the sixth generation (6G) system in terms of drivers, use cases, usage scenarios, requirements, key performance indicators (KPIs), and enabling technologies. First, we attempt to answer the question of "Is there any need for 6G?" by shedding light on the key driving factors of 6G, in which we predict the explosive growth of mobile traffic until 2030 , and envision potential use cases and usage scenarios. Second, the technical requirements of $6 \mathrm{G}$ are discussed and compared with those of $5 \mathrm{G}$ with respect to a set of KPIs in a quantitative manner. Third, the state-of-the-art 6G research efforts and activities from representative institutions and countries are summarized, and a tentative roadmap of definition, specification, standardization, and regulation is projected. Then, we identify a dozen of potential technologies and introduce their principles, advantages, challenges, and open research issues. Finally, the conclusions are drawn to paint a picture of "What 6G may look like?". This survey is intended to serve as an enlightening guideline to spur interests and further investigations for subsequent research and development of $6 \mathrm{G}$ communications systems.

Index Terms-5G, 6G, artificial intelligence, blockchain, cell-free MIMO, digital twin, edge computing, holographic-type communications, Internet of Everything, Internet of Things, machine learning, mobile networks, non-terrestrial networks, optical wireless communications, O-RAN, Tactile Internet, Terahertz, visible light communications, wireless communications

\section{INTRODUCTION}

$\mathbf{T}$ HE mobile telecommunication industry stems from the first generation $(1 \mathrm{G})$ analog cellular systems represented by Advanced Mobile Phone System in the United States and Nordic Mobile Telephone in Europe, which firstly offered mobile voice-calling service around the year 1980. Since then, a new generation of mobile communications was introduced to market nearly every ten years. The $1 \mathrm{G}$ analog systems were replaced by the second generation digital cellular networks in around 1990. Despite of several competing systems, the Global System for Mobile Communications known as GSM [1] achieved a great commercial success and allowed more than one billion of the world's population to enjoy the convenience brought by mobile voice, short texting, and low-rate data services. Exploiting a revolutionary technology named Code-Division Multiple Access (CDMA), the third generation (3G) systems [2] represented by WCDMA, CDMA2000, and TD-SCDMA, were developed and firstly deployed in 2001 to support high-speed data access with a rate of several megabits per second. In December 2009, the commercial Long Term Evolution (LTE) networks [3] were launched in the Scandinavian capitals Stockholm and Oslo, providing the world's first fourth generation (4G) mobile broadband service. The $4 \mathrm{G}$ system that is empowered by a genius combination of multi-input multi-output (MIMO) and orthogonal frequencydivision multiplexing (OFDM) spurs the proliferation of smart phones, fostering the mobile Internet industry that is worth trillions of dollars a year.

W. Jiang (Corresponding author) and H. D. Schotten are with the Intelligent Networking Research Group, German Research Center for Artificial Intelligence (DFKI), 67663 Kaiserslautern, Germany (e-mails: \{wei.jiang, hans_dieter.schotten\}@ dfki.de).

B. Han, M. A. Habibi, and H. D. Schotten are with the Division of Wireless Communications and Radio Navigation (WICON), Department of Electrical and Computer Engineering, University of Kaiserslautern, 67663 Kaiserslautern, Germany (e-mails: \{binhan, asif, schotten\}@eit.uni-kl.de).
In April 2019, when South Korea's three mobile operators and U.S. Verizon were arguing with each other about who is the world's first provider of the fifth generation (5G) communication services, we stepped into the era of 5G. In the past two years, the term of $5 \mathrm{G}$ has been remaining one of the hottest buzzwords in news, attracting unprecedented attention from the whole society. It even went beyond the sphere of technology and economy, becoming the focal point of geopolitical tension. Unlike the previous generations that focused merely on improving network capacities, $5 \mathrm{G}$ expands mobile communication services from human to things, and also from consumers to vertical industries. The potential scale of mobile subscription is substantially enlarged from merely billions of the world's population to almost countless interconnectivity among human, machines, and things. It enables a wide variety of services from traditional mobile broadband to Industry 4.0, virtual reality, Internet of Things (IoT), and automatic driving [4]. In 2020, the outbreak of the COVID-19 pandemic leads to a dramatic loss of human life worldwide and imposes unprecedented challenges on societal and economic activities. It is also this public health crisis that has highlighted the unique value of a number of applications empowered by $5 \mathrm{G}$, such as remote surgeon, online education, remote working, driver-less vehicles, unmanned delivery, robots, smart healthcare, and autonomous manufacturing.

Currently, 5G is still on its way being deployed across the world, but it is already the time for academia and industry to shift their attention to beyond $5 \mathrm{G}$ or the sixth generation (6G) systems, in order to satisfy the future demands for information and communications technology (ICT) in 2030. Even though discussions are ongoing within the wireless community as to whether there is any need for $6 \mathrm{G}$ or whether counting the generations should be stopped at 5, adopting the Microsoft's approach where Windows 10 is the ultimate version, and even there is an opposition to talking about 6G [5], several 
pioneering works on the next-generation wireless networks have been initiated. A focus group called Technologies for Network 2030 within the International Telecommunication Union Telecommunication (ITU-T) standardization sector was established in July 2018. The group intends to study the capabilities of networks for 2030 and beyond [6], when it is expected to support novel forward-looking scenarios such as holographic-type communications, Tactile Internet, multisense networks, and digital twin. The European Commission initiated to sponsor beyond $5 \mathrm{G}$ research activities, as its recent Horizon 2020 calls - ICT-20 $5 G$ Long Term Evolution and ICT-52 Smart Connectivity beyond $5 G$ - where a batch of pioneer research projects for key $6 \mathrm{G}$ technologies were kicked off at the early beginning of 2020. In Finland, the University of Oulu began ground-breaking $6 \mathrm{G}$ research as part of Academy of Finland's flagship program [7] called 6G-Enabled Wireless Smart Society and Ecosystem (6Genesis), which focuses on several challenging research areas including reliable nearinstant unlimited wireless connectivity, distributed computing and intelligence, as well as materials and antennas to be utilized in future for circuits and devices. Besides, other traditional main players in mobile communications such as the United States, China, Germany, Japan, and South Korea already initiated their $6 \mathrm{G}$ research officially or at least announced their ambition and tentative roadmaps. At this crossroad, an overview of the current state of the art and a vision of future communications to provide an enlightening guideline for subsequent research and development works is of interest. Recently, the articles focusing on 6G topics, e.g., use cases, application scenarios, requirements, and promising technological pillars, are emerging in the literature, as summarized in the following subsection.

\section{A. State-of-the-Art Related Works}

The earliest article [8] that discusses the topic of $6 \mathrm{G}$ is published in September 2018, where David and Berndt try to address the question of "Is there any need for beyond $5 \mathrm{G}$ ?" by reviewing the key services and innovations from the $1 \mathrm{G}$ analog system to the virtualized and software-defined 5G infrastructure. Nawaz et al. [9] check the state-of-theart advances in the fields of machine learning (ML) and quantum computing, and then envisage their synergies with communication networks to be considered in the $6 \mathrm{G}$ system. In [10], Rappaport et al. describe the challenges and potentials of Terahertz (Thertz) communications in the development and implementation of $6 \mathrm{G}$ networks. Later, the authors of [11] provide a brief description of vision and potential techniques. In [12], Letaief et al. discuss potential technologies to enable ubiquitous Artificial Intelligence (AI) service in 6G networks (i.e., networking for $\mathrm{AI}$ ) and AI-enabled methodologies for the design and optimization of $6 \mathrm{G}$ (i.e., AI for networking). Zong et al. propose two candidate system architectures for $6 \mathrm{G}$ in [13] and identify several $6 \mathrm{G}$ technologies including photonics-defined radio, holography, and AI. In the context, the authors of [14] aim to shed light on requirements, network architecture, key technologies, and new applications in the upcoming $6 \mathrm{G}$ system. In [15], Strinati et al. envision five technology enablers for $6 \mathrm{G}$, i.e., pervasive AI at network edge, three-dimensional (3D) coverage consisting of terrestrial networks, aerial platforms, and satellite constellation, a new physical layer incorporating sub- $\mathrm{THz}$ and Visible Light Communications (VLC), distributed security mechanisms, and a new architecture. Huang et al. survey the new architectural changes associated with green $6 \mathrm{G}$ networks in [16], which also briefly introduces several potential technologies such as ubiquitous $3 \mathrm{D}$ coverage, pervasive $\mathrm{AI}, \mathrm{THz}, \mathrm{VLC}$, and blockchain. In [17], Jiang and Luo provides a comprehensive and highly coherent treatment on all the technology aspects related to ML for wireless communications and networks.

Since the beginning of 2020, the number of publications related to $6 \mathrm{G}$ grows a bit faster than that of the past two years. In [18], Dong et al. argue that $6 \mathrm{G}$ should be human centric, and therefore security, secrecy, and privacy are key features. To support this vision, a systematic framework, required technologies, and anticipated challenges are outlined. Then, a survey on various ML technologies applied for communication, networking, and security aspects of vehicular networks, and a vision of the ways toward an intelligent 6G vehicular network are provided in [19]. Polese et al. foresee several possible $6 \mathrm{G}$ use cases and present a number of technologies, which are considered by the authors as the enablers for these use cases [20]. Viswanathan and Mogensen attempt to paint a broad picture of communication needs and technologies in the era of $6 \mathrm{G}$ [21], where new themes that are likely to shape $6 \mathrm{G}$ requirements are presented. Zhang, Xiang, and $\mathrm{Xu}$ argue in their article [22] that 1000 times price reduction from the customer's view point is the key to success for the $6 \mathrm{G}$ system. In [23], Chen et al. contribute a comprehensive discussion that covers visions, requirements, technology trends, and challenges, aiming at clarifying the ways to tackle the challenges of coverage, capacity, data rate, and mobility of $6 \mathrm{G}$ mobile communication systems. The authors of [24] share their viewpoints in terms of applications, technological trends, service classes, and requirements, and then give their identification on enabling technologies and open research problems. Kato et al. [25] recognize possible challenges and potential research directions of advancing ML technologies into the future $6 \mathrm{G}$ network from the perspectives of communication, networking, and computing. Guo outlines the core concepts of explainable AI for $6 \mathrm{G}$ in [26], including public and legal motivation, definition, the trade-off between explainability and performance, explainable methods, and an explainable AI framework for future wireless systems. A survey paper [27] provides a comprehensive view of $6 \mathrm{G}$ in terms of applications, requirements, challenges, and research directions. Some key technologies such as AI, terahertz, blockchain, three-dimensional networking, and wireless optical communications are briefly introduced. In [28], the authors aim to extend the vision of $5 \mathrm{G}$ to more ambitious scenarios in a more distant future and speculate on the visionary technologies that can provide the step changes needed for enabling $6 \mathrm{G}$. Guan et al. give a brief presentation on various $6 \mathrm{G}$ issues in [29], including core services, use cases, requirements, enabling technologies, architectures, typical use scenarios, challenges, and research directions. The authors 
TABLE I

SUMMARY OF STATE-OF-THE-ART CONTRIBUTIONS RELATED TO 6G COMMUNICATION SYSTEMS

\begin{tabular}{|c|c|c|c|}
\hline Ref. & Public. Time & Topics & Major Contributions \\
\hline 8 & Sept. 2018 & Vision & Reviews the key services and innovations from $1 \mathrm{G}$ to $5 \mathrm{G}$, and provide a vision for $6 \mathrm{G}$. \\
\hline 9 & April 2019 & ML & $\begin{array}{l}\text { Reviews the state-of-the-art advances in ML and quantum computing, and proposes a quantum computing- } \\
\text { assisted ML framework for } 6 \mathrm{G} \text { networks. }\end{array}$ \\
\hline [10] & June 2019 & Terahertz & $\begin{array}{l}\text { Describes the technical challenges and potentials for wireless communications and sensing above } \\
100 \mathrm{GHz} \text {, and present discoveries, approaches, and recent results that will aid in the development and } \\
\text { implementation of } 6 \mathrm{G} \text { networks. }\end{array}$ \\
\hline [11 & July 2019 & Vision & Outlines a number of key technological challenges and the potential solutions associated with $6 \mathrm{G}$. \\
\hline [12] & Aug. 2019 & AI & $\begin{array}{l}\text { Discusses potential technologies for } 6 \mathrm{G} \text { to enable ubiquitous } \mathrm{AI} \text { applications and AI-enabled approaches } \\
\text { for the design and optimization of } 6 \mathrm{G} \text {. }\end{array}$ \\
\hline [13] & Sept. 2019 & Vision & $\begin{array}{l}\text { Proposes two candidate system architectures for } 6 \mathrm{G} \text { and identify several } 6 \mathrm{G} \text { technologies including } \\
\text { photonics-defined radio, holography, and AI. }\end{array}$ \\
\hline [14 & Sept. 2019 & Survey & A survey aiming to identify requirements, network architecture, key technologies, and new applications. \\
\hline [15] & Sept. 2019 & Technologies & $\begin{array}{l}\text { Five technology enablers for } 6 \mathrm{G} \text {, including pervasive AI at network edge, } 3 \mathrm{D} \text { coverage consisting of } \\
\text { terrestrial networks, aerial platforms, and satellite constellation, a new physical layer incorporating sub- } \\
\text { THz and VLC, distributed security mechanisms, and a new architecture. }\end{array}$ \\
\hline [16 & Dec. 2019 & Green $6 \mathrm{G}$ & $\begin{array}{l}\text { A survey on new architectural changes associated with } 6 \mathrm{G} \text { networks and potential technologies such as } \\
\text { ubiquitous 3D coverage, pervasive AI, THz, VLC, and blockchain. }\end{array}$ \\
\hline [17 & Dec. 2019 & AI & $\begin{array}{l}\text { A special issue provides a comprehensive treatment on all the technology aspects related to ML for } \\
\text { wireless communications, covering fading channel, channel coding, physical-layer design, network slicing, } \\
\text { resource management, mobile edge, fog computing, and autonomous network management. }\end{array}$ \\
\hline [18 & Jan. 2020 & Vision & $\begin{array}{l}\text { Argues that } 6 \mathrm{G} \text { should be human-centric, and therefore security, secrecy, and privacy are key features. } \\
\text { To support this vision, a systematic framework, required technologies, and challenges are outlined. }\end{array}$ \\
\hline [19 & Feb. 2020 & Vehicular & $\begin{array}{l}\text { Summarizes various ML technologies that are promising for communication, networking, and security } \\
\text { aspects of vehicular networks, and envisions the ways towards an intelligent } 6 \mathrm{G} \text { vehicular network, } \\
\text { including intelligent radio, network intelligentization, and self-learning. }\end{array}$ \\
\hline [20] & Mar. 2020 & Use cases & $\begin{array}{l}\text { Foresees several possible use cases and key technologies that are considered as the enablers for these } 6 \mathrm{G} \\
\text { use cases. }\end{array}$ \\
\hline 21 & Mar. 2020 & Survey & $\begin{array}{l}\text { New themes including new human-machine interface, ubiquitous computing, multi-sensory data fusion, } \\
\text { and precise sensing and actuation. Then, major technology transformations such as new spectrum, new } \\
\text { architecture, and new security are presented, with the emphasize of AI's potentials. }\end{array}$ \\
\hline [22] & Mar. 2020 & AI & $\begin{array}{l}\text { Argues that } 1000 \text { times price reduction from the customer's view point is the key to success and uses } \\
\text { AI-assisted intelligent communications to illustrate the drive-force behind. }\end{array}$ \\
\hline 23 & April 2020 & Survey & $\begin{array}{l}\text { A comprehensive discussion of } 6 \mathrm{G} \text { based on the review of } 5 \mathrm{G} \text { developments, covering visions, } \\
\text { requirements, technology trends, and challenges, aiming at clarifying the ways to tackle the challenges } \\
\text { of coverage, capacity, data rate, and mobility of } 6 \mathrm{G} \text { communication systems. }\end{array}$ \\
\hline 24 & May 2020 & Survey & $\begin{array}{l}\text { A vision on } 6 \mathrm{G} \text { in terms of applications, technological trends, service classes, and requirements, as well } \\
\text { as an identification on enabling technologies and open research problems. }\end{array}$ \\
\hline 25 & June 2020 & ML & $\begin{array}{l}\text { Possible challenges and potential research directions of advancing ML technologies into the future } 6 \mathrm{G} \\
\text { network in terms of communication, networking, and computing perspective. }\end{array}$ \\
\hline [26] & June 2020 & AI & $\begin{array}{l}\text { The core concepts of explainable AI for } 6 \mathrm{G} \text {, including public and legal motivations, definitions, the trade- } \\
\text { off between explainability and performance, explainable methods, and an explainable AI framework for } \\
\text { future wireless systems. }\end{array}$ \\
\hline 27 & Aug. 2020 & Survey & $\begin{array}{l}\text { A survey on 6G in terms of applications, requirements, challenges, and research directions. Some key } \\
\text { technologies such as AI, THz, blockchain, and wireless optical communications are briefly introduced. }\end{array}$ \\
\hline 28 & Aug. 2020 & Vision & $\begin{array}{l}\text { Extends the vision of } 5 \mathrm{G} \text { to more ambitious scenarios in a more distant future and speculates on the } \\
\text { visionary technologies that can provide the step changes needed for enabling } 6 \mathrm{G} \text {. }\end{array}$ \\
\hline 29 & Oct. 2020 & Survey & $\begin{array}{l}\text { A brief presentation of various } 6 \mathrm{G} \text { issues including core services, use cases, requirements, enabling } \\
\text { technologies, architectures, typical use scenarios, challenges, and research directions. }\end{array}$ \\
\hline 30 & Oct. 2020 & MIMO & $\begin{array}{l}\text { An overview of holographic MIMO surface (HMIMOS) communications including the available hardware } \\
\text { architectures for re-configuring such surfaces, highlighting the opportunities and key challenges in } \\
\text { designing HMIMOS-enabled wireless communications for } 6 \mathrm{G} \text {. }\end{array}$ \\
\hline 31 & Oct. 2020 & Survey & $\begin{array}{l}\text { A comprehensive study of } 6 \mathrm{G} \text { visions, requirements, challenges, and open research issues, outlining } \\
\text { seven disruptive technologies, i.e., millimeter wave (mmWave) communications, THz communications, } \\
\text { optical wireless communications, programmable metasurfaces, drone-based communications, backscatter } \\
\text { communications, and Tactile Internet. }\end{array}$ \\
\hline
\end{tabular}

of [30] provide an overview of holographic MIMO surface communications as a promising technological enabler for $6 \mathrm{G}$ wireless communications. Recently, Bariah et al. give a comprehensive $6 \mathrm{G}$ vision in [31], identifying seven disruptive technologies, associated requirements, challenges, and open research issues.

\section{B. Contributions}

It is noticed that a comprehensive survey that can provide a complete overview across up-to-date vision, driving forces, 
potential use cases and usage scenarios, requirements, and enabling technologies of $6 \mathrm{G}$, a summary of the state-of-theart $6 \mathrm{G}$ research activities across the world, and a projection of a roadmap for research, specification, standardization, and development toward 2030, is still missing in the literature. The authors of this article also realize that some interesting use cases, applications, and key technologies are not covered by the previous $6 \mathrm{G}$ articles. To fill this gap, we attempt to provide a complete envision of $6 \mathrm{G}$ with respect to drivers, requirements, efforts, and enablers. The main contributions of this article can be listed as follows:

1) It provides a thorough investigation on currentlyavailable publications related to the discussion of $6 \mathrm{G}$.

2) It attempts to answer the question of "Do we really need 6G?" by shedding light on its key drivers, including the explosive growth of mobile traffic and mobile subscriptions until 2030, disruptive use cases, and advanced usage scenarios beyond 5G. It goes beyond the state of the art by identifying the consensus of previous works on use cases and also bringing missing use cases that are of interest, and defining three novel usage scenarios.

3) It discusses the technical requirements of $6 \mathrm{G}$ in terms of a set of key performance indicators (KPIs), which are compared with the KPIs of $5 \mathrm{G}$, if applicable.

4) It summarizes the ambitions, efforts, and research activities on $6 \mathrm{G}$ across the world, while a tentative roadmap of definition, specification, standardization, and regulation is envisaged. To the best knowledge of the authors, that is the first time in the literature.

5) It gives a complete view of potential $6 \mathrm{G}$ technological enablers, which are categorized into several groups: new spectrum consisting of mmWave, $\mathrm{THz}$ communications, VLC, optical wireless communications (OWC), and dynamic spectrum management (DSM), new networking that covers softwarization and virtualization, radio access network (RAN) slicing, open-RAN (O-RAN), and security, new air interface including massive MIMO, intelligent reflecting surfaces (IRS), coordinated multipoint (CoMP), cell-free massive MIMO, and new modulation techniques, new architecture providing 3D coverage by means of integrating large-scale satellite constellation, high-altitude platform (HAP), and unmanned aerial vehicle (UAV) with traditional terrestrial networks, and new paradigm empowered by the convergence of computing-communication resources and the integration of mobile networks, AI, blockchain, and digital twin.

\section{Organization}

The rest of this article is organized as follows: Section II clarifies the key driving forces for the necessity of developing $6 \mathrm{G}$, including the explosive growth of mobile traffic and mobile subscriptions, disruptive use cases, and advanced usage scenarios. Section III analyzes the technical requirements for the $6 \mathrm{G}$ system in terms of a number of KPIs. The ambitions and efforts from the main players in the mobile communication industry are summarized and a development roadmap is estimated in Section IV. Section V provides a complete view of a dozen of key technologies for 6G. Finally, Section VI concludes this article by painting a picture about what $6 \mathrm{G}$ may look like.

\section{DRIVERS}

Since the middle of 2019, commercial 5G mobile networks have been rolled out across the world and already reached a very large scale in some countries. For example, the number of deployed 5G base stations in China exceeds 500000 at the end of 2020, serving more than 100 million 5G subscribers. Following the tradition that a new generation appears every one decade, it is time for both academia and industry to initiate the exploration of the successor of 5G. On the road towards $6 \mathrm{G}$, however, the first problem we encounter is that there are many concerns like "Do we really need $6 G$ ?" or "Is $5 G$ already enough?". To address such questions, we first need to clarify the key driving forces for $6 \mathrm{G}$.

The development of a next-generation system is driven by not only the exponential growth of mobile traffic and mobile subscriptions but also new disruptive services and applications on the horizon. In addition, it is also driven by the intrinsic need of mobile communication society to continuously improve network efficiencies namely cost efficiency, energy efficiency, spectrum efficiency, and operational efficiency. With the advent of advanced technologies such as AI, THz, and large-scale satellite constellation, the communication network is able to evolve towards a more powerful and more efficient system to better fulfil the requirements of current services and open the possibility for offering disruptive services that have hitherto never been seen. In this section, we intend to shed light on three drivers: $i$ ) the explosive growth of mobile traffic, ii) disruptive use cases, and iii) novel usage scenarios. Its technological drivers will be discussed in detail in Section $\mathrm{V}$

\section{A. Explosively-growing Mobile Traffic}

We are in an unprecedented era where a large number of smart products, interactive services, and intelligent applications emerge and evolve in a prompt manner, imposing a huge demand on mobile communications. It can be foreseen that the $5 \mathrm{G}$ system is hard to accommodate the tremendous volume of mobile traffic in 2030 and beyond. Due to the proliferation of rich-video applications, enhanced screen resolution, machineto-machine (M2M) communications, mobile cloud services, etc., the global mobile traffic will continuously increase in an explosive manner, up to $5016 \mathrm{~EB}^{1}$ per month in the year of 2030 compared with $62 \mathrm{~EB}$ per month in 2020, according to the estimation made by ITU Radiocommunication (ITUR) [32] in 2015. A report from Ericsson [33] reveals that the global mobile traffic has reached $33 \mathrm{~EB}$ per month at the end of 2019, which justifies the correctness of ITU-R's estimation.

On the one hand, the number of smartphones and tablets will further increase while other devices such as wearable electronics grows in a faster pace, amounting to a total of 17.1 billion mobile broadband (MBB) users in 2030, as shown in Fig. 1. On the other hand, the traffic demand per MBB user

\footnotetext{
${ }^{1} 1$ Exabyte $(\mathrm{EB})=1000000$ Terabytes $(\mathrm{TB}), 1 \mathrm{~TB}=1000$ Gigabytes $(\mathrm{GB})$
} 
continuously raises in the company of the rising number of MBB users. That is mainly because the popularity of mobile video services such as Youtube, Netflix, and more recently TikTok, as well as the stable improvement of screen resolution on mobile devices. The traffic coming from mobile video services already account for two thirds of all mobile traffic nowadays [33] and is estimated to be more dominant in the future. In some developed countries, a strong traffic growth before 2025 is driven by rich-video services and a long-term growth wave will continue due to the penetration of augmented reality (AR) and virtual reality (VR) applications. The average data consumption for every mobile user per month, as illustrated in Fig. 1. will increase from around $5 \mathrm{~GB}$ in 2020 to over $250 \mathrm{~GB}$ in 2030. In addition to human-centric communications, the scale of M2M terminals will increase more rapidly and will become saturated no earlier than 2030. It is predicted that the number of M2M subscriptions will reach 97 billion, around 14 times over that of 2020 [32]. This serves as another driving force for the explosive growth of mobile traffic.
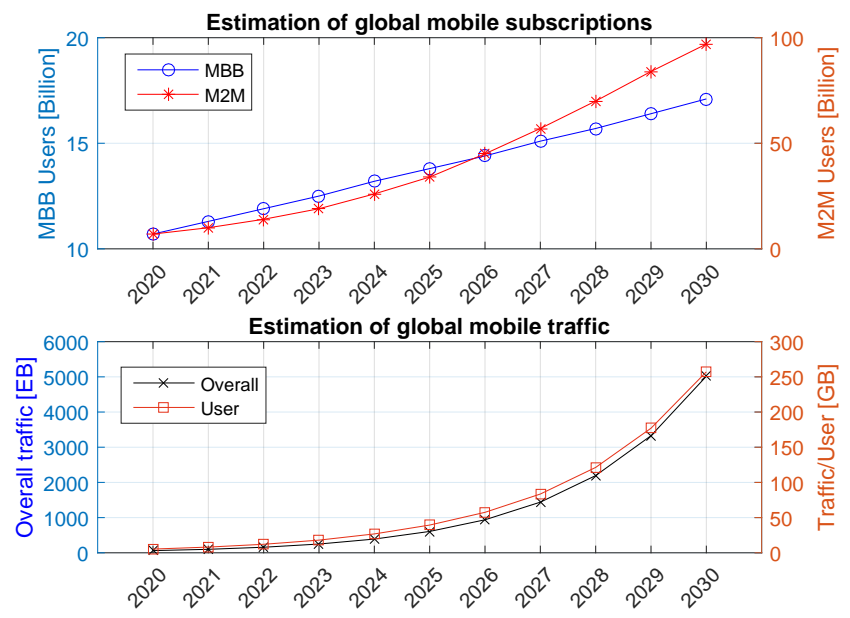

Fig. 1. The estimated global mobile subscriptions and mobile traffic from 2020 to 2030. Source: ITU-R Report M.2370-0 [32].

\section{B. Potential Use Cases}

With the advent of new technologies and continuous evolution of existing technologies, e.g., holography, robotics, microelectronics, photo-electronics, AI, and space technology, many unprecedented applications can be fostered in mobile networks. To explicitly highlight the unique characteristics and define technical requirements of $6 \mathrm{G}$, we foresee several representative use cases as follows:

Holographic-Type Communication (HTC): Compared to traditional 3D videos using binocular parallax, true holograms can satisfy all visual cues of observing 3D objects by the naked eye as natural as possible. With a significant advance of holographic display technology in recent years such as Microsoft's HoloLens [34], it is envisioned that its application will become a reality in the next decade. Remote rendering high-definition holograms through a mobile network will bring truly immersive experience. For example, holographic telepresence will allow remote participants to be projected as holograms into a meeting room or allow the attendee of online training or education to interact with ultra-realistic objects. However, HTC leads to huge bandwidth demands on the order of terabits per second even with image compression. In addition to consider the frame rate, resolution, and color depth in two-dimensional (2D) video, the quality of hologram also involves the volumetric data such as tilt, angle, and position. If representing an object with images every $0.3^{\circ}$, an imagebased hologram with $30^{\circ}$ field of view and a tilt of $10^{\circ}$ needs a 2D array of 3300 separate images [35]. HTC also requires ultra-low latency for true immersiveness and high-precision synchronization across massive bundles of interrelated streams for reconstructing holograms.

Extended Reality (ER): Combining augmented, virtual, and mixed realities, ER starts stepping into practical applications in the age of 5G, but it is still in its infancy analogue to the video service at the beginning of mobile Internet. To achieve the same level of image quality, ER devices with $360^{\circ}$ field of view need much higher data throughput in comparison to $2 \mathrm{D}$ video streaming. For an ideal immersion experience, the quality of video with higher resolution, higher frame rate, more color depth, and high dynamic range are required, leading to a bandwidth demand of over 1.6 Gbps per device [36]. Similar to video traffic that saturates the $4 \mathrm{G}$ networks, the proliferation of ER devices will be blocked by the limited capacity of $5 \mathrm{G}$ with the peak rate of $20 \mathrm{Gbps}$, especially at the cell edge. In addition to bandwidth, interactive ER applications such as immersive gaming, remote surgery, and remote industrial control, low latency and high reliability are mandatory.

Tactile Internet: It provides extremely low end-to-end (E2E) latency to satisfy the 1-millisecond (ms) or less reaction time reaching the limitation of human sense [37]. In combination with high reliability, high availability, high security, and sometimes high throughput, a wide range of disruptive real-time applications are enabled. It will play a critical role in the field of real-time monitoring and remote industrial management for Industry 4.0 and Smart Grid. For example, with immersive audio-visual feeds provided by ER or HTC streaming, together with haptic sensing data, a human operator can remotely control the machinery in a place surrounded by biological or chemical hazards, as well as remote robotic surgery carried out by doctors hundreds of miles away [38]. The typical closedloop controlling, especially for devices or machinery moving rapidly, is very time-sensitive, where an E2E latency below $1 \mathrm{~ms}$ is expected.

Multi-Sense Experience: Human has five senses (sight, hearing, touch, smell, and taste) to perceive external environment, whereas current communications focus only on optical (text, image, and video) and acoustic (audio, voice, and music). The involvement of the senses of taste and smell can create fullyimmersive experience, which may bring some new services for example in food and texture industries [6]. Furthermore, the application of haptic communication will play a more important role and will raise a wide range of applications such as remote surgery, remote controlling, and immersive gaming, etc. This use case brings a stringent requirement on low latency. 
Digital Twin is considered one of the emerging technologies of $6 \mathrm{G}$ communication system, which is used to create a comprehensive and detailed virtual copy of a physical (a.k.a. real) object. The softwarized copy is equipped with a wide range of characteristics, information, and properties related to the original object. Such a twin is then used to manufacture multiple copies of an object with full automation and intelligence. The early rollouts of digital twin has attracted significant attention of a number vertical industries and manufacturers. However, its full deployment is expected to be realized with the development of $6 \mathrm{G}$ networks.

Pervasive Intelligence: With the proliferation of mobile smart devices and the emergence of new-style connected equipment such as robots, smart cars, drones, and VR glasses, over-the-air intelligent services are envisioned to boom. These intelligent tasks mainly rely on traditional computationintensive AI technologies: computer vision, simultaneous localization and mapping (SLAM), face and speech recognition, natural language processing, motion control, to name a few. To overcome stringent computation, storage, power supply, and privacy constrains on mobile devices, $6 \mathrm{G}$ networks will offer pervasive intelligence in an AI-as-a-Service manner [12] by utilizing distributed computing resources across the cloud, mobile edge, and end-devices, and cultivating communicationefficient ML training and interference mechanisms. For example, a humanoid robot such as Atlas from Boston Dynamics [39] is possible to off-load its computational load for SLAM towards edge computing resources, in order to improve motion accuracy, prolong battery life, and become more lightweight by removing some embedded computing components. In addition to computation-intensive tasks, pervasive intelligence also facilitate time-sensitive AI tasks to avoid the latency constraint of cloud computing when fast decisions or responses to conditions are required.

Intelligent Transport and Logistics: In 2030 and beyond, millions of autonomous vehicles and drones provide a safe, efficient, and green movement of people and goods. Connected autonomous vehicles have stringent requirements on reliability and latency to guarantee the safety of passengers and pedestrians. Unmanned aerial vehicles, especially swarm of drones, open the possibility for a wide variety of unprecedented applications, while bringing also disruptive requirements for mobile networks.

Enhanced On-Board Communications: With the development of economy, the activity sphere of human and the frequency of their movement will rapidly increase in the next decade. The number of passengers travelling by commercial planes, helicopters, high-speed trains, cruise ships, and other vehicles, will be very huge, bringing skyrocketing demands on high-quality communication services on board. Despite the efforts in the previous generations until $5 \mathrm{G}$, it is undeniable that on-board connectivity is far from satisfactory in most cases due to high mobility, frequent handover, sparse coverage of terrestrial networks, and limited bandwidth and high cost of satellite communications. Relying on reusable space launching technologies and massive production of satellites, the deployment of large-scale satellite constellation such as SpaceX's Starlink [40] becomes a reality, enabling cost-efficient and

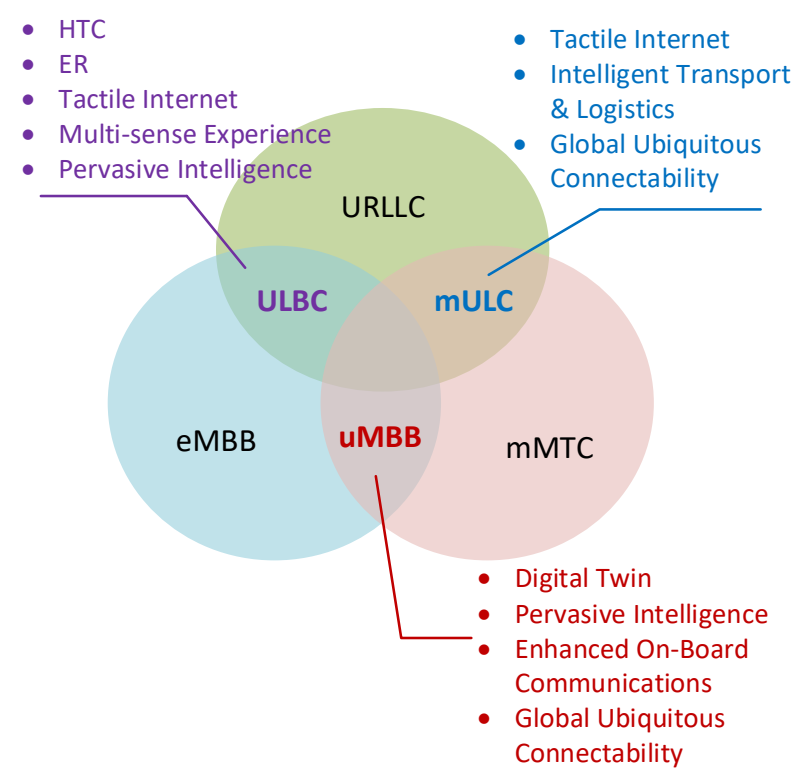

Fig. 2. In addition to typical 5G usage scenarios (eMBB, ULRRC, and mMTC), three enhanced scenarios named uMBB, ULBC, and mULC are proposed by the authors of this article for the $6 \mathrm{G}$ system in order to support disruptive use cases and applications.

high-throughput global coverage. Keep this in mind, $6 \mathrm{G}$ is expected to be an integrated system of terrestrial networks, satellite constellation, and other aerial platforms to provide seamless 3D coverage, which offers high-quality, low-cost, and global-roaming on-broad communication services.

Global Ubiquitous Connectability: The previous generations of mobile communications focused mainly on the dense metropolitan areas, especially indoor scenarios. However, a large population in remote, sparse, and rural areas even have no access to basic ICT services, digging a big digital divide among human around the world. Besides, more than $70 \%$ of the Earth's surface is covered by water, where the growth of maritime applications require network coverage for both water surface and underwater. However, ubiquitous coverage across the whole planet with sufficient capacity, acceptable quality of service (QoS), and affordable cost is far from a reality. On the one hand, it is technically impossible for terrestrial networks to cover remote areas and extreme topographies such as ocean, desert, and high maintain areas, while it is too costly to offer terrestrial communication services for sparsely-populated areas. On the other hand, geostationary earth orbit (GEO) satellites are expensive to deploy and their capacity is limited to several Gbps per satellite [41], which is dedicated only for high-end users such as maritime and aeronautic industries. As mentioned above, the deployment of large-scale low Earth orbit (LEO) satellite constellation will enable low-cost and high-throughput global communication services [42]. The 6G system is envisioned to make use of the synergy of terrestrial networks, satellite constellation, and other aerial platforms to realize ubiquitous connectability for global MBB users and wide-area IoT applications. 


\section{Usage Scenarios}

To define 5G, three usage scenarios have been firstly recommended by ITU Radiocommunication (ITU-R) section M.2083 in 2015 [43]:

- enhanced mobile broad-band (eMBB) addresses the human-centric applications for a high-data-rate access to mobile services, multi-media content, and data. This scenario fosters new services and applications over smart devices (smartphones, tablets, and wearable electronics). It emphasizes wide-area coverage to provide seamless access and high capacity in hot spots.

- ultra-reliable low-latency communications (URLLC) is a disruptive promotion over the previous generation systems that focus on human users. It opens the possibility for mission-critical connectivity for new applications such as automatic vehicles, Smart Grid, and Industry 4.0, which have stringent requirements on reliability, latency, and availability.

- massive machine-type communications (mMTC) supports dense connectivity with a very large number of connected devices typically deployed in IoT scenarios. The devices such as sensors are low-cost, low-power consumption but typically transmitting a low volume of delay-tolerate data.

It can be seen that these $5 \mathrm{G}$ usage scenarios cannot satisfy the technical requirements of the aforementioned $6 \mathrm{G}$ use cases. For instance, an user wearing a lightweight VR glass playing interactively-immersive games requires not only ultrahigh bandwidth but also low latency. Autonomous vehicles on the road or flying drones need ubiquitous connectivity with high throughput, high reliability, and low latency. Through extending the scope of current usage scenarios, as shown in Fig. 2. we propose three enhanced scenarios to cover their overlapping areas. To support high-quality on-board communications and global ubiquitous connectability, the MBB service should be available across the whole surface of the Earth in the era of $6 \mathrm{G}$, called ubiquitous $\mathrm{MBB}$ or $\mathrm{uMBB}$. In addition to its ubiquitousness, another enhancement of $\mathrm{uMBB}$ is a remarkable boost of network capacity and transmission rate for hot spots so as to support disruptive services, e.g., a group of users wearing lightweight VR glasses gathering in a small room where a data rate of several Gbps per user is needed. The uMBB scenario will be the foundation of digital twin, pervasive intelligence, enhanced on-board communications, and global ubiquitous connectability, as the mapping relationship shown in Fig. 2 Ultra-reliable Low-latency Broadband Communication (ULBC) supports the applications requiring not only URLLC but also extreme high throughput such as HTC-based immersive gaming. It is expected that the use cases of HTC, ER, Tactile Internet, multi-sense experience, and pervasive intelligence will benefit from this scenario. Furthermore, the last scenario called massive Ultra-reliable Low-latency Communication (mULC) combines the characteristics of both mMTC and URLLC, which will facilitate the deployment of massive sensors and actuators in vertical industries.

\section{REQUIREMENTS}

To well support disruptive use cases and applications in 2030 and beyond, the 6G system will provide extreme capacity, reliability, efficiency, etc. Like the minimal requirements for International Mobile Telecommunications-2020 (IMT-2020) specified in [44], a number of quantitative or qualitative KPIs are utilized to indicate the technical requirements for $6 \mathrm{G}$. Most of the KPIs that are applied for evaluating $5 \mathrm{G}$ are still valid for $6 \mathrm{G}$ while some new KPIs would be introduced for the assessment of new technological features. The first eight KPIs in the following part are considered as key requirements in the definition of $5 \mathrm{G}$, which are briefly introduced as follows:

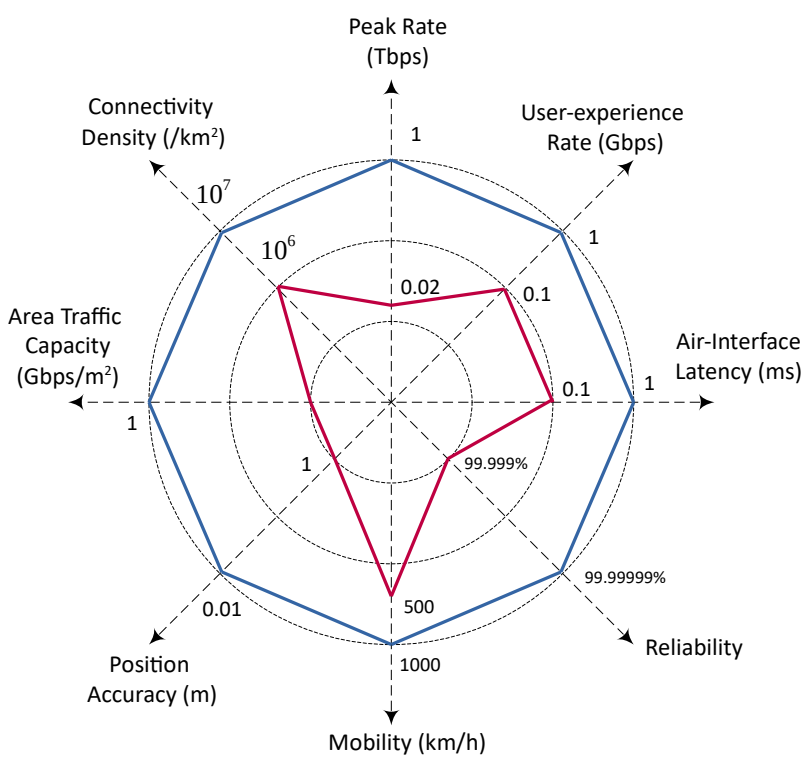

Fig. 3. Comparison of the technical requirements between $5 \mathrm{G}$ and $6 \mathrm{G}$. The vertices of the inner polygon stands for eight key KPIs for 5G, as defined in [44], while the vertices of the outer octagon represent that of $6 \mathrm{G}$.

- Peak data rate is the highest data rate under ideal conditions, in which all available radio resources are totally assigned to a single mobile station. Traditionally, it is the most symbolic parameter to differentiate different generations of mobile systems. Driven by both user demand and technological advances such as $\mathrm{THz}$ communications, it is expected to reach up to $1 \mathrm{Tbps}$, tens of times that of $5 \mathrm{G}$, which has the peak rate of $20 \mathrm{Gbps}$ for downlink and $10 \mathrm{Gbps}$ for uplink.

- User-experienced data rate is defined as the $5^{\text {th }}$ percentile point $(5 \%)$ of the cumulative distribution function of user throughput. In other words, a user can get at least this data rate at any time or location with a possibility of $95 \%$. It is more meaningful to measure the perceived performance, especially at the cell edge, and reflect the quality of network design such as site density, architecture, inter-cell optimization, etc. In the $5 \mathrm{G}$ deployment scenario of dense urban, the target of user-perceived rate is $100 \mathrm{Mbps}$ for downlink and $50 \mathrm{Mbps}$ for uplink. It is expected that $6 \mathrm{G}$ can offer $1 \mathrm{Gbps}$ even higher, which is 10 times that of $5 \mathrm{G}$. 
- Latency can be differentiated into user plane and control plane latency. The former is the time delay induced in a radio network from a packet being sending out at the source until the destination receives it, assuming a mobile station is in the active state. In $5 \mathrm{G}$, the minimum requirement for user plane latency is $4 \mathrm{~ms}$ for eMBB and $1 \mathrm{~ms}$ for URLLC. This value is envisioned to be further reduced to $10 \mu \mathrm{s}$ to $100 \mu$ s. Control plane latency refers to the transition time from a most "battery efficient" state (e.g., the idle state) to the start of continuous data transfer (e.g., the active state). The minimum latency for control plane should be $10 \mathrm{~ms}$ in $5 \mathrm{G}$ and is expected to be also remarkably improved in 6G. In addition to over-the-air delay, round-trip or E2E delay is more meaningful but also complicated due to the large number of network entities involved. In 6G, the E2E latency may be considered as a whole.

- Mobility means the highest moving speed of a mobile station supported by a network with the provisioning of acceptable Quality of Experience (QoE). To support the deployment scenario of high-speed trains, the highest mobility supported by $5 \mathrm{G}$ is $500 \mathrm{~km} / \mathrm{h}$. In $6 \mathrm{G}$, the maximal speed of $1000 \mathrm{~km} / \mathrm{h}$ is targeted if commercial airline systems are considered.

- Connection density is the KPI applied for the purpose of evaluation in the usage scenario of mMTC. Given a limited number of radio resources, the minimal number of devices with a relaxed QoS per square kilometer $\left(\mathrm{km}^{2}\right)$ is $10^{6}$ in $5 \mathrm{G}$, which is envisioned to be further improved 10 times to $10^{7}$ per $\mathrm{km}^{2}$.

- Network energy efficiency is important to realize costefficient mobile networks and reduce the total carbon emission for green ICT, playing a critical role from the societal-economic respective. After the early deployment of $5 \mathrm{G}$ networks, there is already some complaints about its high energy consumption although the energy efficiency per bit has been substantially improved in comparison with the previous generations. In $6 \mathrm{G}$ networks, this KPI would be $10-100$ times better over that of $5 \mathrm{G}$ so as to improve the energy efficiency per bit while reducing the overall power consumption of the mobile industry.

- Peak spectral efficiency is an important KPI to measure the advance of radio transmission technologies. The minimum requirement in $5 \mathrm{G}$ for peak spectral efficiencies are $30 \mathrm{bps} / \mathrm{Hz}$ in the downlink and $15 \mathrm{bps} / \mathrm{Hz}$ in the uplink. Following the empirical data, it is expected that advanced $6 \mathrm{G}$ raido technologies can achieve three times higher spectral efficiency over the 5G system.

- Area traffic capacity is a measurement of the total mobile traffic that a network can accommodate per unit area, relating to the available bandwidth, spectrum efficiency, and network densification. The minimal requirement for $5 \mathrm{G}$ is $10 \mathrm{Mbps}$ per square meter $\left(\mathrm{m}^{2}\right)$, which is expected to reach $1 \mathrm{Gbps} / \mathrm{m}^{2}$ in some deployment scenarios such as indoor hot spots.

In addition to the aforementioned key capabilities, there are several extended or novel KPIs may be also required to evaluate the requirements of $6 \mathrm{G}$.

- Reliability relates to the capability of transmitting a given amount of traffic within a predetermined time duration with high success probability. This requirement is defined for the purpose of evaluation in the usage scenario of URLLC. In 5G networks, the minimum requirement for the reliability is measured by a success probability of $1-$ $10^{-5}$ when transmitting a data packet of 32 bytes within $1 \mathrm{~ms}$ given the channel quality of coverage edge for the deployment scenario of urban macro environment. It is expected to improve at least two orders of magnitude, i.e., $1-10^{-7}$ or $99.99999 \%$ in the next-generation system.

- Signal Bandwidth is the maximum aggregated system bandwidth. The bandwidth may be supported by single or multiple RF carriers. The requirement for bandwidth in $5 \mathrm{G}$ is at least $100 \mathrm{MHz}$, and $6 \mathrm{G}$ will support up to $1 \mathrm{GHz}$ for operation in higher frequency bands or even higher in $\mathrm{THz}$ communications or OWC.

- Positioning accuracy of the 5G positioning service is better than $10 \mathrm{~m}$. Higher accuracy of positioning has a strong demand in many vertical and industrial applications, especially in indoor environment that cannot be covered by satellite-base positioning systems. With the application of $\mathrm{THz}$ radio station, which has a strong potential in high-accuracy positioning, the accuracy supported by $6 \mathrm{G}$ networks is expected to reach centimeter (cm) level.

- Coverage in the definition of $5 \mathrm{G}$ requirement mainly focuses on the received quality of radio signal within a single base station. The coupling loss, which is defined as the total long-term channel loss over the link between a terminal and a base station and includes antenna gains, path loss, and shadowing, is utilized to measure the area served by a base station. In $6 \mathrm{G}$ networks, the connotation of coverage should be substantially extended considering that the coverage will be globally ubiquitous and will be shifted from only $2 \mathrm{D}$ in terrestrial networks to $3 \mathrm{D}$ in a terrestrial-satellite-aerial integrated system.

- Timeliness is an emerging time-domain performance requirement to future communication systems. Typical metrics of timeliness include the well-known ageof-information (AoI) [45], and its recently proposed variants such like age-of-task (AoT) [46] and age-ofsynchronization (AoS) [47]. Differing from the classical memoryless metric of latency, which focuses on the overall delay experienced by all data packets or service sessions throughout their delivery process, the concept of timeliness emphasizes the freshness of the latest data and service that are successfully delivered to the end user. This brings to the system an endogenous birth-time discrimination against outdated data/service, as well as a memory to its historical state(s), and therewith raises both the impact and the complexity of task scheduling in system optimization.

\section{ROADMAP AND EFFORTS}

Even though discussions are ongoing within the wireless community as to whether there is any need for $6 \mathrm{G}$ and 


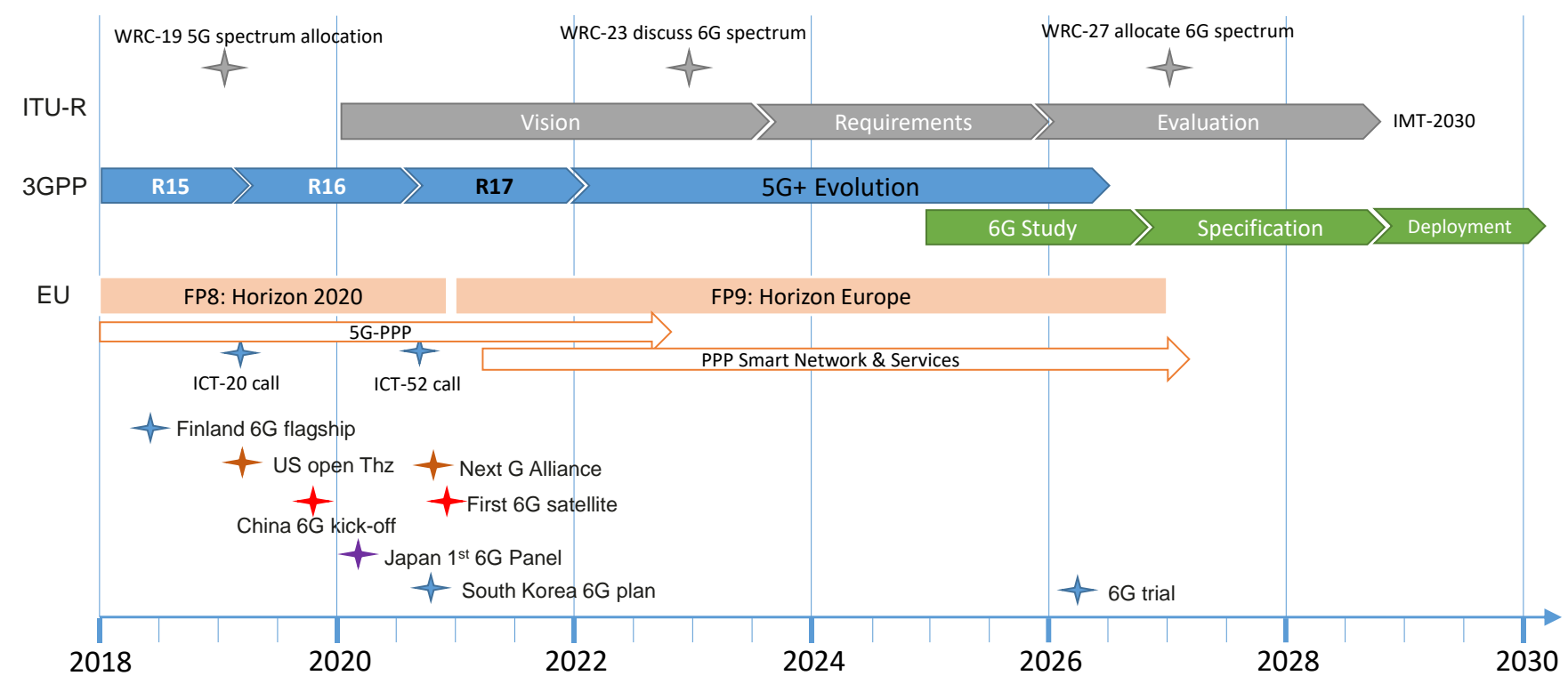

Fig. 4. The predicted roadmap of research, definition, specification, spectrum regulation, development, and deployment for $6 \mathrm{G}$ mobile systems.

whether counting the generations should be stopped at 5 , a few pioneering works on the next-generation wireless networks have been initiated. In July 2018, a focus group "Technologies for Network 2030" was established under ITU-T [6]. This group intends to study and review existing technologies, platforms, and standards for identifying the gaps and challenges towards the capabilities of networks for the year 2030 and beyond, with the emergence of novel forward-looking scenarios such as holographic applications, Tactile Internet, multi-sense networks, and digital twin. Although it mainly focuses on fixed data communication networks, the vision, requirements, architecture, and novel use cases identified in this group also has reference values for the definition of the $6 \mathrm{G}$ mobile system. According to the empirical timeline, ITU-R section will initiate the study of $6 \mathrm{G}$ vision and will publish the requirements for IMT for 2030 (as the requirements of IMT-2020 [44] published in 2017) in the middle of the 2020s, and then will step into the evaluation phase afterwards. ITU-R is also responsible for organizing the world radiocommunication conference (WRC) that governs the frequency assignment, being hold every three to four years. In WRC-19 held in 2019, the spectrum allocation issue for the $5 \mathrm{G}$ system was determined. It is expected that the WRC probably scheduled in 2023 (WRC-23) will discuss the spectrum issues for $6 \mathrm{G}$ and the spectrum allocation for $6 \mathrm{G}$ communications may be formally decided in 2017 (WRC-27).

In the early of 2019, the third generation partnership project (3GPP) has frozen the Release 15 (Rel. 15 or R15) specifications, which are the first phase of 5G standards. Rel. 15 mainly focuses on eMBB and provides the basis for URLLC, especially in respect of the support of low latency. In July 2020, the subsequent release (i.e., Rel. 16) has been completed as the second phase of $5 \mathrm{G}$ standards [48]. In addition to enhancements for the existing Rel. 15 features, new features such as non-public network, new radio (NR) unlicensed, NR positioning, NR-Light, and integrated access and backhaul
(IAB) have been introduced so as to fully support URLLC and industrial IoT. Currently, a more advanced version (Rel. 17) is being standardized by 3GPP and is expected to be completed in the early of 2021, in spite of a delay due to the COVID-19 pandemic. Driven by a multitude of key stakeholders from the traditional mobile industry, a wide range of verticals, and the non-terrestrial industry, it is envisioned as the most versatile release in 3GPP history in terms of features content, including NR over non-terrestrial networks, NR beyond $52.6 \mathrm{MHz}$, NR sidelink enhancement, network automation, etc. 3GPP is expected to standardize several subsequent releases to further evolve the $5 \mathrm{G}$ system, which can be called $5 \mathrm{G}+$ or $5 \mathrm{G}$ Evolution. According to the experiences got in previous generations, $6 \mathrm{G}$ will be a disruptive system that cannot be developed following such a backward-compatible manner. In parallel, therefore, 3GPP is expected to initiate the study item for $6 \mathrm{G}$ around the year 2025 , followed by the phase of specification, to guarantee the first commercial deployment roll-out of 6G by 2030 .

In October 2018, the European Commission has initiated to sponsor beyond $5 \mathrm{G}$ research activities by opening the ICT20-2019 call "5G Long Term Evolution" under the eighth Framework Programmes for Research and Technological Development (FP8) being named Horizon 2020. Eight projects such as 5G-COMPLETE [49] and 5G-CLARITY [50] have been selected from a total of 66 proposals and kicked off in early 2020. In its recent call ICT-52-2020 "Smart Connectivity beyond $5 G$ ", the accepted projects selected from a high-competitive evaluation process explicitly shown that their ambition is to provide the early research efforts on 6G. In its upcoming research and innovation framework program called Horizon Europe or FP9, a large number of efforts and funding will concentrate on the research and development of 6G and will be organized in the framework of Public Private Partnership (PPP) "Smart Network \& Services", following the 
successful strategy of the 5G Infrastructure Public Private Partnership (5G-PPP) under Horizon 2020.

Besides, many countries have announced and are implementing ambitious plans to launch $6 \mathrm{G}$ research and development initiatives. In Finland, the University of Oulu began ground-breaking 6G research as part of Academy of Finland's flagship program [7] called 6G-Enabled Wireless Smart Society and Ecosystem (6Genesis), which focuses on several challenging research areas including reliable nearinstant unlimited wireless connectivity, distributed computing and intelligence, as well as materials and antennas to be utilized in future for circuits and devices. As early as 2016, the U.S. Defense Advanced Research Projects Agency (DARPA), along with companies from the semiconductor and defense industries, has initiated the joint university microelectronic project (JUMP), among which the center for converged $\mathrm{THz}$ communications and sensing seeks to develop technologies for a future cellular infrastructure. In March 2019, the US spectrum regulator - the Federal Communications Commission (FCC) announced to open up experimental licences for the use of frequencies between $95 \mathrm{GHz}$ and $3 \mathrm{THz}$ for $6 \mathrm{G}$ and beyond. In October 2020, the Alliance for Telecommunications Industry Solutions (ATIS) announced the launch of the "Next $G$ Alliance" [51], an industry initiative that aims to advance North American mobile technology leadership in 6G over the next decade. Its ambition it to encompass the full lifecycle of $6 \mathrm{G}$ research and development, manufacturing, standardization, and market readiness. The founding members include AT\&T, T-Mobile, Verizon, Qualcomm, Ericsson, Nokia, Apple, Samsung, Google, Facebook, Microsoft, etc. In November 2019, China has officially kicked off the $6 \mathrm{G}$ technology research and development works coordinated by the Ministry of Science and Technology, together with five other ministries or national institutions. A promotion working group from government that is in charge of management and coordination, and an overall expert group that is composed of 37 experts from universities, research institutes, and industry were established at this event. Later, it was announced that China aims to form $6 \mathrm{G}$ overall development ideas by the end of 2020 . In November 2020, China launched what it claimed is the first 6G experimental satellite to test communications from space using high-frequency terahertz spectrum. In early 2020, the Japanese government set up a dedicated panel including representatives from the private sector and academia to discuss technological development, potential use cases, and policy. Japan reportedly intends to dedicate around $\$ 2$ billion to encourage privatesector research and development for $6 \mathrm{G}$ technology. In late 2020, the government of South Korea has confirmed a plan to carry out a 6G trial in 2026 and is expected to spend approximately $\$ 169$ million over the course of five years to develop $6 \mathrm{G}$ technology. The trial aims to achieve $1 \mathrm{Tbps}$ in data transmission speeds and latency reduction to one-tenth of current $5 \mathrm{G}$ services. The government will initially push for tasks in six key areas (hyper-performance, hyper-bandwidth, hyper-precision, hyper-space, hyper-intelligence, and hypertrust) to preemptively secure next-generation technology.

\section{TECHNOLOGICAL ENABLERS}

To well support disruptive use cases and applications, advanced technologies on transmission, networking, and computing would be developed and then applied in the $6 \mathrm{G}$ system. This section provides a complete view of potential $6 \mathrm{G}$ technological enablers, which are categorized into several groups: new spectrum consisting of mmWave, $\mathrm{THz}$ communications, VLC, OWC, and DSM, new networking that covers softwarization and virtualization, RAN slicing, O-RAN, and security, new air interface including massive MIMO, IRS, CoMP, cellfree massive MIMO, and new modulation techniques, new architecture providing 3D coverage by means of integrating large-scale satellite constellation, HAP, and UAV with traditional terrestrial networks, and new paradigm empowered by the convergence of communication, computing, and storage resources, as well as the integration of AI, blockchain, digital twin, and mobile networks. The principle, advantages, challenges, and open research issues for each identified technology are introduced.

\section{A. New Spectrum}

Next generation cellular networks provide a good capability of heterogeneous radio access technology (RAT), where the legacy RAT with low radio frequencies and the line-of-sight (LOS)-dependent RATs (THz, VLC, and OWC) can co-exist well. THz, VLC, and OWC may construct a new layer in the hierarchical RAN architecture (e.g., picocells), where heterogeneous cells with different RAT are overlaying on each other. The ideology is similar to the introduction of mmWave in the $5 \mathrm{G}$ networks.

\section{1) Millimeter Wave}

The mmWave technology has been introduced by the $5 \mathrm{G}$ new radio, and believed to remain as an essential component in future $6 \mathrm{G}$ networks. Compared to legacy radio frequency (RF) technologies working below $6 \mathrm{GHz}$, it significantly broadens the available bandwidth with new carrier frequencies up to $300 \mathrm{GHz}$. Such a huge new bandwidth, as Shannon's theorem reveals, will inflate the radio channel capacity and quench the imminent thirsty for higher data rate. Meanwhile, the shorter wavelength also leads to smaller antenna size. This not only improves the portability and integration level of device, but also allows to increase the dimension of antenna arrays and therewith narrow the beams [52], which is beneficial to specific applications such like detection radars and physical layer security. Furthermore, the atmospheric and molecular absorption exhibit highly variant characteristics at different frequencies across the mmWave band, providing potential for diverse use cases. On the one hand, low attenuation can be observed at some special bands such as $35 \mathrm{GHz}, 94 \mathrm{GHz}$, $140 \mathrm{GHz}$, and $220 \mathrm{GHz}$, making long-distance peer-to-peer communications possible at these frequencies; on the other hand, severe propagation loss is experienced at some "attenuation peaks" such as $60 \mathrm{GHz}, 120 \mathrm{GHz}$, and $180 \mathrm{GHz}$, which can be exploited by short-range covert networks with stringent safety requirements [52], [53]. Currently, the standardization efforts in the mmWave field are mainly focusing on the 
TABLE II

CATEGORIZED KEY TECHNOLOGY ENABLERS WITH ADVANTAGES AND CHALLENGES

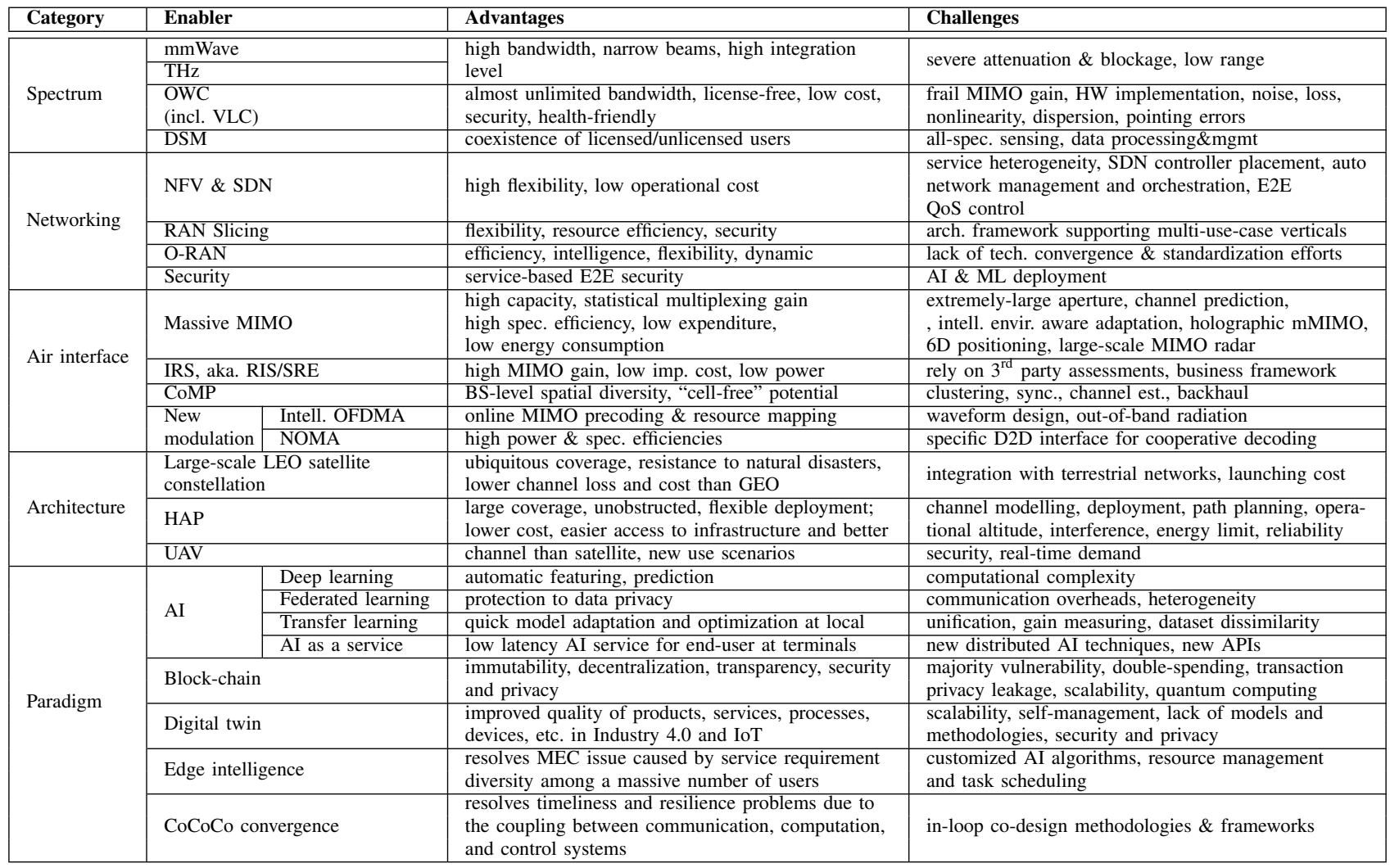

$60 \mathrm{GHz}$ band for indoor use, e.g. the ECMA-387 [54], the IEEE 802.15.3c [55], and the IEEE 802.11ad [56].

Accompanying to the benefits of mmWave technologies, there also come new challenges. First of all, the broad bandwidth in mmWave band and high transmission power can lead to severe non-linear signal distortions, which proposes higher technical requirements for the integrated circuits than those for RF devices. Meanwhile, since the effective transmission range of mmWave, especially in the $60 \mathrm{GHz}$ band, is severely limited by the atmospheric and molecular absorption, mmWave channels are commonly dominated by the LOS path. This becomes a major drawback that is further magnified by the poor diffraction at this short wavelength, which causes a strong blockage loss in scenarios with dense presence of small-scale obstacles such as vehicles, pedestrians, or even the human body of user itself [57]. The high propagation loss and LOS-dependency also significantly raises the channel state sensitivity to the mobility, i.e. the impact of fading is much stronger than that in the RF bands. The demand for an outstanding mobility management becomes therefore unprecedentedly high. Furthermore, in scenarios with dense links coexisting, especially for indoor environments, the interference among different access points will be significant, interference management approaches are therefore called for [58].

\section{2) Terahertz Communications}

Despite of its current abundance in spectral redundancy, mmWave is hardly adequate to tackle down the increasing cravenness on bandwidth for another decade. Looking forward to the $6 \mathrm{G}$ era, wireless technologies operating at even higher frequencies, such as $\mathrm{THz}$ or optical frequency bands, are expected to play an important role in the next generation RAN, providing extremely high bandwidth.

Similar to mmWave, $\mathrm{THz}$ waves also suffer from high path loss and therefore highly rely on directive antennas and LOS channels while providing a very limited coverage. However, when a satisfactory LOS link is available, the high carrier frequency brings a bandwidth that is significantly higher than any legacy technology, which makes it possible to simultaneously provide ultra-high performance in aspects of throughput, latency, and reliability. Moreover, compared to both mmWave systems working at lower frequencies and wireless optical systems working in higher frequency bands, $\mathrm{THz}$ communication systems are insensitive to atmospheric effects, which eases the tasks of beamforming and beam tracking. This shapes $\mathrm{THz}$ communication into a good supplementary solution in addition to the mainstream RF technologies for specific use cases, such as indoor communications and wireless backhaul; and a competitive option for future cyber-physical applications with extreme QoS requirements, such like real-time VR/AR [59].

Furthermore, the high carrier frequency also allows smaller antenna size for higher integration level. It is expected [14] that over 10000 antennas can be embedded into a single $\mathrm{THz}$ BS to provide hundreds of super-narrow beams simultaneously, so as to overcome the high propagation loss, and to simultaneously achieve extremely high traffic capacity together with massive connectivity, which assemble to unlock 
its applications in ultra-massive machine-type communications such as Internet-of-Everything (IoE) [60].

Nevertheless, while $\mathrm{THz}$ outperforms mmWave in many ways, it also faces stronger technical challenges, especially from the aspect of implementing essential hardware circuits, including antennas [61], amplifiers [62], and modulators. Especially, it has been since long the most critical challenge for practical deployment of $\mathrm{THz}$ technologies, to efficiently modulate baseband signals onto such high frequency carriers with integrated circuits. To address this issue, a great effort has been made over the past decade, leading to a prosperous set of developments, most of which are solid state $\mathrm{THz}$ systems that rely on frequency mixing, such as the one reported in [63]. Recently, it has also been discussed to apply spatial direct modulation in $\mathrm{THz}$ systems, so as to directly modulate baseband signals to $\mathrm{THz}$ band without any intermediate frequency stage.

\section{3) Visible Light Communications}

VLC works in the frequency range of $400 \mathrm{THz}$ to $800 \mathrm{THz}$. Differing from the RF technologies in lower $\mathrm{THz}$ range that use antennas, VLC relies on illumination sources - especially light-emitting diodes (LEDs) - and image-sensor or photodiode arrays to implement the transceivers. With these transceivers, a high bandwidth can be easily achieved with low power consumption (100 $\mathrm{mW}$ for $10 \mathrm{Mbps}$ to $100 \mathrm{Mbps}$ ) without generating electromagnetic or radio interference [64]. The good power efficiency, the long lifetime (up to 10 years) and low cost of mainstream LEDs, in addition to the unlicensed access to spectrum, makes VLC an attractive solution for use cases sensitive to battery life and access cost, such like massive IoT and wireless sensor network (WSN). Moreover, VLC also exhibits better propagation performance than RF technologies do in some non-terrestrial scenarios, such as aerospace and underwater, which can be important part of the future 6G ecosystem, as we will introduce later in Sec. V-D.

Compared to RF, the MIMO gain in VLC, especially in indoor scenarios, is very frail. This roots in the high coherence among the propagation paths, i.e. the low spatial diversity. Though this coherence can be somehow reduced by using spaced LED arrays [65], MIMO-VLC is also challenged by the design and implementation of receivers: non-imaging receivers are extremely sensitive to their spatial alignments with the transmitters, while imaging receivers are not applicable in cost-critical use cases for their high prices [66]. Therefore, there has been so far no MIMO method standardized into the mainstream VLC physical layer of IEEE 802.15.7, despite of the persistent efforts made in academia since a decade [67], [68], [69]. Therefore, the beamforming in VLC, differing from the MIMO-based RF beamforming, is implemented by a special optical device known as spatial light modulator (SLM) [70].

Similar to mmWave and $\mathrm{THz}$ technologies, VLC also relies on LOS channels, since it has neither ability to penetrate, nor sufficient diffraction to bypass common obstacles. Meanwhile, due to the concerns about adjacent cell interference and almost ubiquitous environment light noise, VLC systems generally require directive antennas with narrow beams. These facts make VLC systems highly sensitive to the position and mobility of users, leading to a high requirement of beam tracking. On the other hand, this feature can be also exploited for advantages in certain use scenarios, such like better accuracy in indoor positioning [71], and lower interference in vehicular communications [72].

Another key technical challenge for VLC roots in the open and unregulated (to be more specific, unregulatable) access to visible light spectrum, which implies a higher security risk and calls for a more stringent security requirement in VLC systems, when compared to legacy cellular systems in licensed RF bands. Regarding this, physical layer security has been extensively investigated as a promising solution [73].

\section{4) Optical Wireless Communications}

OWC points to wireless communications [74] that use infrared (IR), visible light, or ultraviolet (UV) as transmission medium. It is a promising complementary technology for traditional wireless communications operating over RF bands. OWC systems operating in the visible band are commonly referred to as VLC, which attracts much attention recently and is discussed separately in Sec. V-A3 The optical band can provide almost unlimited bandwidth without the permission from the regulators worldwide. It can be applied to realize high-speed access at low cost thanks to the availability of optical emitters and detectors. Since the IR and UV waves have a similar behavior as the visible light, the security risks and interference can be significantly confined and the concern about the radio radiation to human health can be eliminated. It is expected to have obvious advantages in deployment scenarios such as vehicular communications in smart transportation systems, airplane passenger lighting, and medical machines that are sensitive to electromagnetic interference. Despite its advantage, OWC suffers from the impairments such as ambient light noise, atmospheric loss, nonlinearity of LEDs, multi-path dispersion, and pointing errors [75].

In OWC, LED or laser diode (LD) is applied to convert an electrical signal to an optical signal at the transmitter and the receiver uses a photodiode (PD) to convert the optical signal into electrical current. The information is conveyed by modulating the intensity of optical pulse simply through widely-used scheme such as on-off keying or pulse-position modulation, as well as advanced multi-carrier schemes [76] such as OFDM to get higher transmission rate. To support multiple users in a single optical access point, OWC can apply not only typical electrical multiplexing technologies such as time-division, frequency-division, and code-division multi-access, but also optical multiplexing such as wavelengthdivision multi-access. Optical MIMO technology [77] is also implemented in OWC, where multiple LEDs and multiple PDs are applied, in contrast to the multiple antennas in a typical MIMO system operating in the RF band.

The optical system applying image sensors to detect the optical pulse also called optical camera system. The imagine sensor can convert the optical signal into the electrical signal, which has the advantage of easier implementation due to the wide spread of camera-embedded smart phones. On the other hand, terrestrial point-to-point OWC also known as free-space optical (FSO) communications [78] take place at the near IR band. Using high-power high-concentrated laser 
beam at the transmitter, the FSO system can achieve high data rate, i.e., $10 \mathrm{Gbps}$ per wavelength, over long-distance (up to $10000 \mathrm{~km}$ ). It offers a cost-effective solution for the backhaul bottleneck in terrestrial networks, enables crosslinks among space, air, and groud platforms, and facilitates highcapacity inter-satellite links for the emerging LEO satellite constellation. Furthermore, there has also been a growing interest on ultraviolet communication [79] as a result of recent progress in solid state optical transmitter and detector for nonLOS UV communications that offer broad coverage and high security.

\section{5) Dynamic Spectrum Management}

Alongside the continuous mining of unused spectrum at ever-higher frequencies, there is a second approach towards our vision of bandwidth prosperity in 6G: to improve the radio resource utilization rate by DSM.

The idea of DSM dates back to the well-known listenbefore-talk (LBT) protocol applied in IEEE 802.11, which treats all users equally in a contention-based control of access to the spectrum, and one can exploit a band only when it is not occupied by the others. In the unlicensed industrial, scientific and medical (ISM) band, LBT has demonstrated a great success in collision and interference control. Meanwhile, in the licensed spectrum, as reported by the FCC of the U.S., it is the under-utilization of spectrum caused by regulated access "a more significant problem than the physical scarcity of spectrum" [80]. This fact has raised intense research interest on the topic of LBT-like dynamic spectrum sharing among various systems with heterogeneous RATs and different priorities to access licensed/unlicensed bands. Driven by the successful development of software-defined radio technologies, these research efforts gave birth to the technology of cognitive radio (CR) [81], which became rapidly mature in the first decade of this century. Since the LTE era, it has become a distinguished topic in the field of wireless networks to study the DSM in coexistence of licensed cellular systems and unlicensed ISMband technologies [82].

Regarding the future 6G systems, the demand for DSM is becoming unprecedentedly imperative. On the one hand, radio access to ISM bands (especially the IEEE 802.11 bands) is becoming nowadays almost an standard functionality of mainstream cellular terminals, making it a universal solution to provide extra network capacity in scenarios with dense users. On the other hand, since it is impossible to reserve the broad bands of $6 \mathrm{G}$ new spectrum for licensed use only (saliently the visible light spectrum), 6G system is foreseen to suffer in the unlicensed part of its spectrum from the ubiquitous existence of interference by other systems and environmental noises, which are highly dynamic and environment-dependent. 6G systems therefore must be able to dynamically and cognitively select the most appropriate operating band with respect to the instantaneous situation.

There are numerous technical challenges that 6G DSM is facing. In perspective of the hardware implementation, it is mainly troubled by the broadness of $6 \mathrm{G}$ new spectrum that leads to a difficulty in designing transceivers capable of dynamic all-spectrum sensing [83]. The 6G front-end must be capable to carry out a rapid and power-efficient spectrum sensing across the ultra-wide $6 \mathrm{G}$ bands, so as to enable an online radio environment cognition and a timely adaptation to spectrum access. On the system level, for better efficiency and safty of the DSM, the physical layer CR based on spectrum sensing shall be further completed by an awareness of cyberphysical level context information, in order to obtain a deeper understanding in the communication environment, including terrain scenario, traffic pattern, local regulation, etc. This leads to challenges in various aspects of context-awareness, ranging from data provisioning to data ownership [84]

\section{B. New Networking}

To deploy the aforementioned use cases utilizing the discussed key enabling technologies, the network infrastructure is expected to be flexible, intelligent, and open to multi-vendor equipment and multi-tenancy. To that aim, softwarization and virtualization of $6 \mathrm{G}$ network is the first and foremost step. Based on such architecture, the network slicing and resource isolation in both core and RAN domains are considered as novel mechanisms to decrease complexity in the management and orchestration operations. These aspects, along with their privacy and security concerns, of $6 \mathrm{G}$ mobile networks are discussed in this subsection.

\section{1) Softwarization and Virtualization}

Most of the network functions of the $5 \mathrm{G}$ core network (5GC) and next-generation RAN (NG-RAN) architecture are virutalized using network function virtualization (NFV) technology, which provides high flexibility in order to adapt to various scenarios, requirements, and use cases of next generation communication systems [85]. In practice, however, NFV faces some critical challenges that are required to be solved, such as the increased amount of virtual network functions (VNFs), the various requirements of the different tenants, the resources orchestration of the VNFs in a shared infrastructure, the complexity in management and orchestration operations, and others [86]. Therefore, the allocation of virtual network resources, the management and orchestration of the VNFs in a multi-tenant environment require cuttingedge tools and promising solutions to be addressed, such as the AI techniques [87] and ML algorithms [88]. In this context, the European Telecommunication Standards Institute (ETSI) Industry Specification Group (ISG) introduced the Experiential Network Intelligence (ENI) working group in order to improve the experience of network operators and add value to the teleco provided services [89]. The main objectives of the ENI is to exploit $\mathrm{AI}$ and $\mathrm{ML}$ techniques in order to adjust the VNFs of the networked services based on dynamic changes in the requirements of the end users, the conditions of the environments, and the goals of the business. The current specification of the ENI is in its initial phase. Further work is needed to explore the deployment of the AI and ML in the $\mathrm{NFV}$ of the beyond $5 \mathrm{G}$ and $6 \mathrm{G}$ mobile networks.

The software-defined networking (SDN) is considered as one of the most critical key enablers of 5G mobile networks, integrated with NFV capabilities, they are able to offer high flexibility in network management and achieve high effectiveness in service modularity [85]. Based on its integral role and 
performance in the $5 \mathrm{G}$ mobile network, the $\mathrm{SDN}$ is going to be indispensable for the evolution of beyond $5 \mathrm{G}$ and $6 \mathrm{G}$ mobile networks as well and will continue to play a crucial role within their management, orchestration, architectures, etc. [12]. Despite its numerous advantages in 5G, the SDN technology has a number of key research problems that are needed to be addressed in order to thwart full exploitation of its potentials in the future generation of communication networks. These key challenges that continue to confront SDN in the wake of $6 \mathrm{G}$ include, but not limited to: effectively resolving the optimal placement of the SDN controller in the future networks [90], [91], efficiently maintaining the end-to-end and timely global views of the dynamic network topology and its links states [92], exploiting AI/ML techniques for automated network management [93] and orchestration [94], and traffic engineering with guaranteed stringent QoS requirements of heterogeneous services [95].

\section{2) RAN Slicing}

Slicing the RAN architecture, using SDN and NFV technologies, is an emerging research direction towards cloudification, virtualization, and centralization of RAN resources in beyond $5 \mathrm{G}$ and $6 \mathrm{G}$ mobile networks [96]. The slicing-aware NG-RAN architecture helps mobile operators to efficiently slice the entire infrastructure (or a part of it) based on the requirements of end users and vertical industries [97]. Such a classification is also applicable in terms of slicing the NGRAN into eMBB, URLLC, and mMTC subnets. The resources required by these three types of RAN slices are divided into physical and virtual resources. The physical resources are managed by the 3GPP network slicing management system [98] whereas the virtual resources are managed by the ETSI network function virtualization-management and orchestration (NFV-MANO) [99].

Towards an efficient RAN slicing, currently, some of the radio processing functionalities of a next-generation NodeB (gNB) in the NG-RAN are accommodated as VNFs [100], namely the centralized unit (CU) and distributed unit (DU), and some others are distributed as physical network function (PNF), namely the radio unit (RU). The VNFs run on points of presence (PoPs) [101], while the PNFs are implemented on dedicated hardware in the cellular network sites. In the next generation of mobile networks, the full virtualization of $\mathrm{CU}$ and DU, and the partial/full virtualization of RU, can lead to a number of advantages, such as increased performance of the RAN architecture, deployment of RAN slice subnets, decreased network expenditure, simplified operations and management of the network, etc. [102]. We, thus, firmly believe that further studies are needed to address the virtualization of RU functionalities towards a virtualized and slicing-aware RAN of the $6 \mathrm{G}$ mobile networks [103].

Focusing on the function split in the NG-RAN, the current distribution of the radio processing functions over gNB components is executed statically without taking into consideration the type of RAN slices. When supporting a large amount RAN slices in types of eMBB, URLLC, and mMTC, the one-size-fits-all function splitting architecture in NG-RAN is not efficient in terms of resource allocation and network performance [100]. We strongly believe that a customized and dynamic distribution of the gNB functionalities is needed in order to satisfy the service requirements of the three aforementioned types of RAN slices in future networks. Such a dynamic distribution of gNB functionalities shall improve the utilization of physical and virtual resources, enhance the NGRAN performance, and maintain a significant level of isolation and customization among RAN slices of different types while considering the metrics of service level agreements between the mobile operator and tenants [104].

The NG-RAN is expected to support a massive amount of RAN slice subnets. Each type of RAN slice subnet fulfills the service requirements of a singe type of use case of a single tenant. Nevertheless, there is a large number of vertical industries - such as automobile, manufacturing, power grid, and others - which are consisting of multiple use cases [102]. Providing RAN slice subnets for multiple use cases consisting of vertical industries is a crucial research problem that is needed to be addressed in the next generation of mobile networks. One of the crucial parts of this research problem is to design an extensive architectural framework for the $6 \mathrm{G}$ RAN domain in order to support the RAN slice subnets of multi-use-case verticals. This management and orchestration framework shall effectively manage the CU, DU, and RU of per-vertical per-use-case RAN slices in 6G RAN using AI and ML.

\section{3) Open-RAN}

The key concepts of O-RAN, including its vision, architecture, interfaces, technologies, objectives, and other important aspects, were introduced for the first time by the O-RAN alliance in a white paper [105]. The O-RAN alliance has then further studied the use cases leveraging the O-RAN architecture to demonstrate its capability in real time behavior in [106]. The main objective of the openness and intelligence in RAN architecture is to build a radio network that is resource efficient, cost effective, software-driven, virtualized, slicingaware, centralized, open source, open hardware, intelligent, and therefore more flexible and dynamic than any previous generation of mobile networks. To do so, the research community has introduced the utilization of $\mathrm{AI}$ and ML techniques on every single layer of the RAN architecture to fulfill the requirements of dense network edge in beyond $5 \mathrm{G}$ and $6 \mathrm{G}$ mobile communication systems.

By opening up the RAN from a singe vendor environment to a standardized, open, multi-vendor, and $\mathrm{ML}$ and AI powered hierarchical controller structure, it allows third parties and mobile operators to dynamically deploy innovative applications and emerging services that cannot be deployed or supported in legacy RAN architectures. In addition, the ORAN is built upon the NFV-MANO reference architecture proposed by ETSI, which deploys commercial off-the-shelf hardware components, virtualization techniques, and software pieces. The virtual machines abstracted (or virtualized) from the underlying physical resources are easily created, deployed, configured, and decommissioned. Such a virtualized environment and virtual resources, therefore, do not only bring flexibility to the O-RAN architecture, but also decrease the capital/operation expenditures (CAPEX/OPEX) and energy consumption towards $6 \mathrm{G}$ communication networks. 
Despite the flexibility and interoperability that O-RAN offers to mobile operators, it also has a number of key problems that require further research efforts for its full realization in future mobile networks, such as the convergence of different vendors' technologies and various operators on the same platform, the harmonization of different management and orchestration frameworks to deliver enhanced QoE, lacked standards for the validation and troubleshooting of performance issues related to the network, and others. To overcome these challenges, researchers from industry and academia are also expected to take part in theoretical analysis and practical roll-outs of this technology towards an open and intelligent RAN for $6 \mathrm{G}$ mobile networks.

\section{4) Security}

In 5G communication systems, security and privacy are considered as the most critical components for business continuity. This issue has even been raised to the stage of international politics, for example, some countries are proposing sanctions to ban the $5 \mathrm{G}$ network hardware and software of certain vendors, claiming "to protect their network and citizens" from security risks that they concern about with this technology. Focusing on the technical aspect of the $5 \mathrm{G}$ security, together with user equipment, the rise of new business and innovations such as vertical industries, mobile virtual network operators, multi-tenancy, etc. have put a burden on the commercial deployment of 5G networks. For instance, mMTC types of communication services require lightweight security while eMBB and URLLC types of communication services demand high efficient security. Therefore, the $5 \mathrm{G}$ systems (in contrasts with the legacy mobile communication) requires service-based network security to protect its infrastructure from unauthorized access. In such a context, the E2E network slicing solution can be considered an effective mechanisms to secure the $5 \mathrm{G}$ and beyond networks.

To ensure the E2E security for $6 \mathrm{G}$, the deployment of the AI techniques and ML algorithms can play a significant role in the design, implementation, and optimization of security protocols in order to protect network, user equipment, and vertical industries from unauthorized access and threats. With the ongoing evolution in $\mathrm{AI}$ and $\mathrm{ML}$, these technologies will decide the feasibility and implementation of an end to end automation of security protocols. Therefore, further researches are needed to tackle the security challenges and issues that next generation of communication networks are faced with in order to fully automate the network security and privacy with the help of AI systems and ML techniques.

\section{New Air Interface}

The union of OFDM and small-scale active MIMO antenna arrays has ruled the world of cellular radio access network over the entire era of $4 \mathrm{G}$, and is still continuously showing its dominance in recent progresses of 5G. However, the grandiose march towards ever-higher carrier frequency, as we have seen in Sec. $\mathrm{V}$-A , is squeezing the last drop of technical potential from this air interface. Facing new challenges such as high propagation loss and low NLOS path diversity, various emerging technologies are expected to play their key roles in the evolution towards the next generation air interface, which shall be capable to fully exploit the advantages of $6 \mathrm{G}$ new spectrum and support future use cases with extreme performance requirements. This evolution is primarily expected to make several significant shifts and extensions by means of MIMO: from small-scale to massive, from active antenna to passive reflective surface, from physical layer to network layer. New modulation and multiplexing schemes, as complements, are also arising on the horizon of the next decade.

\section{1) Massive $M I M O$}

In legacy cellular networks the MIMO is divided into two types: the point-to-point MIMO and the multi-user MIMO. In the former, multiple antennas are installed in both user equipment and base station, however a single user equipment is served at a single time. In the latter, an antenna array is installed in the base station which provides connection to many user equipment under its respective coverage area. In order to further enhance user experience, increase throughput, and scale up the statistical multiplexing gain, the concept of massive MIMO was introduced in order to address the shortcomings of conventional multi-user MIMO [85]. Since then, the massive MIMO has been considering one of the key enablers of the legacy wireless communication systems. In addition, the massive MIMO is also expected to provide a significant increase in the system capacity, higher statistical multiplexing gain, spectral efficiency, lower CAPEX/OPEX, decreased energy consumption, and many other advantages in beyond $5 \mathrm{G}$ and $6 \mathrm{G}$ cellular networks.

To commercially deploy massive MIMO, many operators have configured base stations with 64 fully digital transceiver chains which have proved its realization in the $5 \mathrm{G}$ mobile networks [107]. These rollouts proved that limitations due to pilot contamination have addressed and for a better spectral efficiency the relevant signal processing methods have been developed and deployed. To pave the way towards the realization of massive MIMO in beyond $5 \mathrm{G}$ and $6 \mathrm{G}$ mobile networks, the authors in [108] and [107] have outlined a number of research challenges including the (i) deployment of extremely large aperture arrays; (ii) limitation in channel prediction; (iii) implementation of intelligent environment aware adaptation; (iv) fundamental limits of wireless communication with holographic Massive MIMO; (v) six-dimensional positioning; and (vi) large-scale MIMO radar. These research problems give a chance to researchers working in academia, industry, and standardization organizations to concentrate their attention towards further improvement in the realization of massive MIMO in next generation of communication networks.

\section{2) Intelligent Reflecting Surfaces}

While releasing a significant bandwidth to support high throughput, the use of high frequency bands over $10 \mathrm{GHz}$ also introduces new challenges, such as higher propagation loss, lower diffraction and more blockage. In the frequency range of mmWave, massive MIMO has been proven effective in realizing active beamforming to provide high antenna gain for overcoming the channel loss. Nevertheless, its capability can be insufficient for the future $6 \mathrm{G}$ new spectrum, as we have discussed earlier in Sec. V-A3 Among all potential candidate solutions to enhance current beamforming approaches, the 
technology of IRS has been widely considered promising for $6 \mathrm{G}$ mobile networks.

The so-called IRS, a.k.a. reconfigurable intelligent surfaces (RIS) [109], is assembled by a category of programmable and reconfigurable material sheets that are capable of adaptively modifying their radio reflecting characteristics. When attached to environmental surfaces, e.g. walls, glass, ceilings, etc., IRS enables to convert parts of the wireless environment into smart reconfigurable reflectors, known as smart radio environment (SRE) [110], and therewith to exploit them for a passive beamforming that can significantly improve the channel gain, at low costs of implementation and power consumption in comparison to active massive MIMO antenna arrays. Moreover, unlike antenna arrays that must be compact enough for integration, SREs are implemented on large-size surfaces apart from the UEs, making it easier for them to realize accurate beamforming with ultra-narrow beams, which are essential for some applications such like physical layer security. Furthermore, unlike active mMIMO antenna arrays that must be specifically implemented for every individual RAT, the passive reflection mechanism that IRS is relying on works almost universal for all RF and optical frequencies, which is especially cost beneficial for the $6 \mathrm{G}$ systems that work in an ultra broad spectrum.

Though IRS is showing a great technical competitiveness in context of the $6 \mathrm{G}$ new spectrum, it still lacks mature techniques for accurate modeling and estimation of the channels and the surface themselves, especially in the near-field range. Moreover, a commercial deployment is only possible after addressing the business concern, that IRS relies on external assessments such like buildings that do not belong to the MNOs. Therefore, it calls for a thoughtful design and standardization of framework providing essential interfaces, agreements, and signaling protocols, so that $6 \mathrm{G}$ operators become capable to widely access and exploit IRS-equipped objects in public and private domains.

\section{3) Coordinated Multi-Point and Cell-Free}

CoMP refers to a class of technologies that allow multiple access points to jointly serve multiple mobile stations, so that a network layer MIMO can be realized to increase the spatial diversity on the top of classical physical layer MIMO approaches. Therefore, it is also known as network MIMO or cooperative MIMO. CoMP was initially introduced by $3 \mathrm{GPP}$ in its Release 11 [111] for LTE Advanced systems. With recent evidences of its potentials in mitigating downlink inter-cell interference and joint user detection in uplink, CoMP is expected to play an important role in $5 \mathrm{G}$ [112]. In the upcoming $6 \mathrm{G}$ era, regarding the new spectrum over $10 \mathrm{GHz}$, the CoMP technologies that make use of base station level diversity will be come an important complement to the traditional antennalevel spatial diversity, as the latter can be minimized by the dense blockage phenomena in high-frequency bands.

Furthermore, since CoMP is generally suggesting every UE to simultaneously hold multiple links to different access points (even when they are of the same RAT), it reveals the feasibility of a novel "cell-free" RAN architecture, where numerous single-antenna access points distributed over the coverage area are connected to a central processing unit, and jointly serving all UEs by coherent transmission in a CoMP fashion [113]. Recent study has shown that such cell-free massive MIMO are able to outperform traditional cellular massive MIMO while also reducing the fronthaul signaling [114].

As a trade-off for the performance gain originated from its nature of cooperative decoding, CoMP also has to face some key technical challenges caused by the same reason. First of all, the performance of CoMP highly relies on the clustering of cooperating base stations, so an appropriate clustering scheme must be found, which has been a focus of research over the past years [115]. Second, the synchronization among cooperating base stations have to be accomplished without inter-carrier and inter-symbol interference [116]. The channel estimation and equalization also must be carried out in a interBS-coherent manner, which greatly increases the computation complexity.

\section{4) New Modulation}

The 3GPP LTE-Advanced (LTE-A) networks are implemented based on orthogonal frequency division multiple access (OFDMA) [117], which is a typical instance of orthogonal multiple access (OMA) technologies prohibiting physical resource block (PRB) sharing by multiple users. In comparison to CDMA, which is deployed in $3 \mathrm{G}$ systems, OFDMA shows a conspicuous superiority in combating multi-path fading by simple and robust carrier-based channel equalization. Furthermore, when combined with MIMO, OFDMA is capable to overwhelmingly outperform CDMA in spectral efficiency. Nevertheless, the full performance of MIMO-OFDM highly relies on the MIMO precoding and resource mapping, which have to be precisely adapted to the channel condition to achieve the optimum. As the dimension of MIMO increase, from up to $8 \times 4$ in LTE-A gradually to over $256 \times 32$ massive MIMO, and eventually to the future ultra-massive MIMO (e.g. $1024 \times 64)$, the complexity of MIMO-OFDM adaptation is dramatically increasing. Meanwhile, in response to the demand of supporting higher mobility - which implies higher fading dynamics - the computation latency constraint to this online adaptation procedure is also becoming more and more strict. To cope with these emerging challenges, a new architecture of AI-driven MIMO-OFDM transceivers has been proposed towards future $6 \mathrm{G}$ systems, which relies on $\mathrm{AI}$ techniques to efficiently solve the problem of online MIMO precoding and resource mapping [14].

Alongside with a further evolution in OFDMA technologies, non-orthogonal multiple access (NOMA) technologies are also widely considered as an answer to the new challenges in the next generation of mobile communication networks. In contrast to OMA, NOMA allow multiple users to reuse the same PRB, which can be achieved by complex inter-user interference cancellation. NOMA approaches can be generally divided into two categories, namely the power-domain (PD) NOMA and the code-domain NOMA. While PD-NOMA has been recently proposed and is attracting a lot of research interest in context of 5G [118], code-domain NOMA has a longer history in legacy systems (e.g. CDMA in 3G), and provides an alternative to PD-NOMA with numerous variations, such as trellis-coded multiple access (TCMA) [119], interleave division multiple access (IDMA) [120], multi- 
user shared access (MUSA) [121], pattern-division multiple access (PDMA) [122], and sparse-code multiple access (SCMA) [123].

Since the beyond $5 \mathrm{G}$ and the $6 \mathrm{G}$ networks are expected to simultaneously manage a massive amount of links, e.g. in the mMTC scenario and its future extensions, NOMA solutions appear promising since they provide higher bandwidth efficiency than OMA approaches. Recent studies have also demonstrated that NOMA can be effectively exploited in new spectrum, including mmWave, $\mathrm{THz}$, and optical frequencies. Additionally, when deployed together with CoMP, NOMA has been proven as capable to outperform CoMP-OMA in both power efficiency and spectral efficiency.

Being completely based on successive interference cancellation, NOMA has a significantly higher complexity in its receiver design than OMA, which increases in polynomial or even exponential order along with the number of users. Especially, in some scenarios that require cooperative decoding across different UEs, specific D2D interfaces must be reserved for this functionality, and security/trust concerns shall be taken into account, to enable the deployment of NOMA in 6G.

\section{New Architecture}

So far, all legacy and existing cellular systems have been designed to substantially rely on terrestrial base stations. For marine, oceanic, as well as wild terrestrial areas, which are impossible or economically challenging to be covered by terrestrial cellular networks, satellites have been since long the most common communication solution. Aiming at a better coverage rate, deployment of non-terrestrial infrastructures as part of the 6G network is being treated as an emerging topic, known as the integrated space and terrestrial network (ISTN). An ISTN is expected to consist of three layers: the groundbased layer constructed by terrestrial base stations, the airborne layer empowered by HAP and UAV, and the spaceborne layer implemented by satellites.

\section{1) Large-Scale Satellite Constellation}

Until now, the coverage of terrestrial networks has only reached a small portion of the whole surface of the globe. First, it is technically impossible to install terrestrial base stations for offering large-scale coverage in ocean and desert [41]. Second, it is difficult to cover extreme topographies, e.g., high maintain area, valley, and cliff, while it is not cost-efficient to use a terrestrial network to provide services for sparsely-populated areas. Additionally, terrestrial networks are vulnerable to natural disasters such as earthquake, flood, hurricane, and tsunami, where there is a vital demand of communications but the infrastructure is destroyed or in outage of service. With the expansion of human activity, e.g., the passengers in commercial planes and cruise ships, the demand of MBB services in uncovered areas increasingly grows. Also, the connectivity demand of IoT deployment scenarios like wild environmental monitoring, Offshore wind farm, and smart grid require wide-area ubiquitous coverage. Satellite communications have been since long the most common solution for wide coverage but currently the mobile communication service offered by GEO satellites is costly, low data rate, and high latency due to the expensive cost for launching and its wide area coverage ( $1 / 3$ surface of earth per GEO satellite).

The satellites in LEO [42] have some advantages over GEO satellite for providing communication services. A LEO satellite operates in an orbit generally lower than $1000 \mathrm{~km}$, which can substantially lower the latency due to the signal propagation compared to the GEO satellite in the orbit of $36000 \mathrm{~km}$. Meanwhile, the propagation loss of LEO is much smaller, facilitating the direction connectivity to mobile and IoT devices that are strictly constraint by the battery supply. Moreover, a stationary ground terminal like an IoT device mounted in a monitoring position may suffer from an obstacle in the line of sight from GEO. There is an early attempt to implement a global satellite mobile communication system, i.e., the Iridium constellation [124] that became commercially available in November 1998. It consists of 66 LEO satellites at an altitude of approximately 781 kilometers and provides mobile phone and data services over the entire Earth surface. Even though it fails due to expensive costs and lack of demands at that time, it is a great technological breakthrough. It still operates today and the second generation Iridium system has been successfully deployed last year.

In recent years, the high-tech company SpaceX gains a lot of attention due to its revolutionary development of space launching technologies. Its reusable rocket namely Falcon 9 dramatically lower the cost of space launching, opening the possibility for deploying large space infrastructures. In January 2015, SpaceX announced its ambition called Starlink [125], utilizing a very large-scale constellation with thousands of LEO satellites to provide global Internet access services [40]. The U.S. FCC has approved its first-stage plan to launch 12000 satellites and another application for deploying 30000 additional satellites is under consideration. With the advancement of electronic technology, the weight of each satellite reduces to approximately $260 \mathrm{~kg}$ and a compact, flat-panel design minimizes the volume, allowing for a dense launch of 60 Starlink satellites to make full use of the capability of Falcon 9. Each satellite becomes cheap due to the massive production, in combination with reusable rockets, building a largescale constellation becomes feasible from both commercial and technological perspectives. Since its first launch in May 2019, around 900 satellites have been successfully deployed through 15 times launches and the speed of deployment can reach 120 satellites per month. In October 2020, SpaceX started to invite some early users to join public testing and it was reported that data rate vary from $50 \mathrm{Mbit} / \mathrm{s}$ to $150 \mathrm{Mbit} / \mathrm{s}$ and latency from $20 \mathrm{~ms}$ to $40 \mathrm{~ms}$ can be expected. Considering its announced monthly fee of 99 USD for active services and its steadily improvement of capacity and performance with the increasing number of deployed satellites, its impact on terrestrial networks should be seriously taken into account. Looking forward to a future global ubiquitous converge that is available anywhere and anytime, it is strongly suggested to integrate satellite networks into the $6 \mathrm{G}$ network as part of it.

\section{2) High Altitude Platform}

In general, terrestrial networks and satellite communications are two technologies that dominate mobile communications for long years. HAP, a quasi-stationary aerial platform operating in 
the stratosphere at an altitude between $17 \mathrm{~km}$ to $22 \mathrm{~km}$ above the Earth's surface represents a new alternative to provide a multitude of telecommunication services in a cost-efficient way [126]. In comparison with terrestrial base stations, HAP can cover a larger area, offer unobstructed connectivity with high signal arrival angle, and provide the flexibility of quick deployment with less temporal and spatial constraints [127]. Compared to satellite systems, HAP has the following advantages: much lower cost of implementation and deployment due to the avoidance of space launching, the possibility of upgrading, repairing, and redeployment, and much shorter propagation distance that corresponds to higher signal strength and lower latency [128].

An aerial platform can carry on-board base stations of both terrestrial and satellite segments, providing connectivity to the terminals of end users transparently. It can keep quasistationary, be redeployed, or be moved from one site to another on demand. This is therefore an efficient solution to improve the coverage of terrestrial and satellite systems. The aerial platform is not only a host of communication services. It well adapts to different applications such as high-definition multimedia broadcasting, remote sensing, surveillance, intelligent transportation, and environmental monitoring. It is also applied for navigation and positioning, working standalone or as a local enhancement component of Global Navigation Satellite System, for a higher accuracy and better availability [129]. Last but not least, it has unique value in local temporary events, emergence communications, and natural disaster (e.g., earthquake, flood, and tsunami) relief, where the terrestrial infrastructure is destroyed or the power grid is in outage of services.

In $6 \mathrm{G}$ systems, the synergy among terrestrial, satellite, and HAP is worth exploring to provide ubiquitous, robust, and resilient network infrastructure and communication connectivity. For example, using HAP to provide high-throughput backhaul links for the small cells deployed in the sites that are hard or expensive to provide wired links, or applying HAP to enhance or relay the satellite signals. From the perspective of the integrated terrestrial-HAP-satellite systems, there are several technical challenges to be solved such as the power supply of the aerial platform, the stability of antenna array, channel models, seamless handover, admission control, interference management, etc. To this end, extensive research on the topics such as 3D channel modeling [130], advanced multi-antenna technologies [131], spectrum-awareness, dynamic spectrum management, and FSO [132] has been carried.

\section{3) Unmanned Aerial Vehicle}

Alongside the space satellite and HAP, UAV also play an indispensable role, as the last piece of the puzzle that fills the gap of near-earth altitude, in the foreseen ISTN. Over the past years, it has been widely discussed to use UAVs in cellular networks as flying BSs or relays [133], [134]. Generally, it makes a flexible mobile supplement to the fixed terrestrial gNBs and space satellites, offering a possibility to dynaically re-plan the RAN by flexibly deploying UAVs to different locations. Compared to HAPs, UAVs are deployed closer to the ground, which not only makes them much cheaper, but also grants them better channel gain with lower path loss.
Especially, upon emergencies (e.g. in disaster reliefs or search $\&$ rescue), UAV also provides a low-cost solution of temporary wireless service delivery to inaccessible areas, such like caves, tunnels, and dilapidated buildings in earthquakes/fires, which cannot be covered by satellites and HAPs.

Furthermore, empowered by the latest achievements of wireless power transmission, UAVs can also be exploited as mobile and automated wireless chargers [135]. Especially, with the simultaneous wireless information and power transfer (SWIPT) technologies, the missions of battery charging and information transmission can be accomplished in a seamless joint [136]. This significantly raises the feasibility of massive deployment of battery-life-critical UEs, which is essential in some emerging use scenarios such as dense WSN. With such rich potentials, UAVs are nowadays widely considered as an essential component of future $6 \mathrm{G}$ infrastructure.

In addition to its irreplaceable position in the $6 \mathrm{G}$ network infrastructure, UAV is also expected to contribute to the prosperity of new use cases and emerging applications in 6G. With its well developed integration with multimedia devices such as video camera and microphone, UAV has since long been widely used in offline photographing and videographing. By nature it is promised to play a larger role in future online video streaming, and can be enabling remote sensing and multi-sense experience when equipped with variant sensors. The high flexibility, mobility and continuously decreasing load cost of UAVs also lead to their foreseeable deployment in future intelligent logistic applications.

\section{E. New Paradigm}

The recent revival of $\mathrm{AI}$ technology spurs the discussions of whether $6 \mathrm{G}$ will be an integrated system of $\mathrm{AI}$ and mobile networks. The $6 \mathrm{G}$ system is expected to support the upsurge of diversified mobile AI applications, and in turn AI will play a critical role in designing and optimizing the wireless architecture [137]. The similar trends are happening in other fields like blockchain and digital twin, which are recognized as strong drivers to shape the next generation mobile system. It is foreseen that $6 \mathrm{G}$ will transform into a huge computer, which converges distributed communication, computing, storage, sensing, and controlling resources for provisioning services of pervasive computing, AI, blockchain, digital twin, etc.

\section{1) Artificial Intelligence}

On the list of $6 \mathrm{G}$ enabling technologies, $\mathrm{AI}$ is recognized as the most potential one. As mobile networks are increasingly complex and heterogeneous, many optimization tasks become intractable, offering an opportunity for advanced ML techniques. Categorized typically into supervised, unsupervised, and reinforcement learning, ML is being considered as a promising data-driven tool to provide computational radio and network intelligence from the physical layer [138] to network management [139]. As a sub-branch of ML, deep learning [140] can mimic biological nervous systems and automatically extract features, extending across all three mentioned learning paradigms. It has a wide variety of applications to against the big challenges in wireless communications and 
networking, being applied to form more adaptive transmission (power, precoder, coding rate, and modulation constellation) in massive MIMO [141], to enable more accurate estimation and prediction of fading channels [142], [143], to provide a more efficient RF design (pre-distortion for power amplifier compensation, beam-forming, and crest-factor reduction), to deliver a better solution for intelligent network management [87], and to offer more efficient orchestration for mobile edge computing, networking slicing, and virtual resources management [88].

In addition to deep learning, a few cutting-edge ML techniques represented by federated learning and transfer learning start showing strong potential in wireless communications. Data-driven methods always have to take into account the issue of data privacy, which limits the manner of processing collected data. In some scenarios, distributing data is strictly prohibited and only local processing on the device where the data was collected is allowed. Federated learning is a method achieving the fulfillment of this requirement by processing the raw data locally and distributing the processed data in a masked form. The mask is designed such that each of the individual data processing expose no information, whereas their cooperation allows for meaningful parameter adjustments towards a universal model. While federated learning gives a method of training ML models from a large number of data sources without ever exposing sensitive data, it creates only one shared model for universal applicability. When individual adjustments of models are required for their deployment to be successful, transfer learning can be used as a tool enabling these adjustments and doing so in a manner requiring a much lower amount of data. By reusing the major part of pre-trained models in a different environment and only adjusting some of the parameters, transfer learning is able to provide quick adaptations using only a low amount of local data.

In addition to using AI to assist with the operation of the networks (i.e., AI for Networking), it is also important to use the ubiquitous computing, connectivity, storage resources to provide mobile AI services to end users in an AI-as-a-Service paradigm (i.e., Networking for AI) [12]. Principally, this provides deep edge resources to enable AI-based computation for new-style terminals such as robots, smart cars, drones, and VR glasses, which demand large amount of computing resources but limited by embedded computing components and power supply. Such AI tasks mainly means the traditional computation-intensive AI tasks, e.g., computer vision, SLAM, speech and facial recognition, natural language processing, and motion control.

\section{2) Blockchain}

With the great success of a kind of cryptocurrency known as Bitcoin, the blockchain technology has received enormous attention in both industry and academia [144]. A blockchain is essentially a distributed public ledger spreading across all participants deployed typically in a peer-to-peer network. A chain of blocks originates from the first block called the genesis block. A new block is appended to the chain via a hash value that is generated according to the information of its parent block. Each block typically consists of two parts: the block header and transaction data. In particular, the header mainly contains the following information: block version indicating the validation rule, the hash of its parent block, timestamp, the number of transactions, and MerkelRoot that concatenates the hash values of all the transactions in this block. A chain continuously grows as blockchain users perform transactions. A miner records and packs a batch of transactions into a block by solving a computationally difficult problem called Proof of Work (PoW). The newly mined block is then broadcasted to the whole blockchain network and all the nodes join the consensus process to validate its trustfulness and update the new block into the chain.

The blockchain has the following technological advantages [145]: Immutability: the transaction data in the blockchain is unchangeable once it is recorded since each block is linked with other blocks via the hash value. The possibility of breaking the whole chain and modifying the content of all blocks is very limited. Decentralization: it applies the consensus mechanisms to manage and maintain the distributed ledge without the need of a centralized entity or third party. The blocks are replicated and shared over an entire blockchain network, thereby avoiding the risk of single point of failure, enhancing data persistency and security, and providing flexibility. Transparency: all blockchain participants have equal right and can access all transaction information of blockchain. Security and privacy: the adoption of asymmetric cryptography, the inherit feature of data immutability, consensus mechanism, and anonymous addressing ensure the security, trustworthiness, and privacy of the blockchain. Despite these promising merits, scalability is a key barrier when the blockchain technology is widely applied from the perspectives of throughput, storage and networking. Enabling technologies [146] related to the number of transactions in each block, block interval time, data transmission, and data storage to realize scalable blockchain systems are recently studied.

Recently, the potential of blockchain in $5 \mathrm{G}$ and beyond systems has been initially investigated in the literature [147]. It is applied to enhance the technologies such as edge computing, NFV, network slicing, and device-to-device communications, to implement important services, e.g., the sharing of spectrum and radio resource, data storage and sharing, network virtualization, security and privacy, in the use case domains of smart city, smart transportation, smart grid, smart healthcare, and UAV. On the other hand, the deployment of $5 \mathrm{G}$ networks can boost the application of blockchain systems. The ubiquitous connectivity, computing, and storage resources provided by mobile networks can be employed to provide local computing power for mobile blockchain systems [148] so as to support solving PoW puzzles, hashing, encryption, and consensus execution. It is envisioned that the blockchain will be converged into the upcoming 6G system for more flexible, secure, and efficient information infrastructure.

\section{3) Digital Twin}

Digital twin is an emerging technology and one significant use case of $6 \mathrm{G}$ communication system. It refers to the logical copy (a.k.a., virtual object or softwarized copy) of a physical object [149]. The virtual representation shall reflect all the dynamics, characteristics, critical components, and important properties of an original physical object that operates and lives 
throughout its life cycle [150]. The digital twin is following the life cycle of a physical twin. Therefore, its monitoring, controlling, maintenance, prediction, and optimization processes are started and ended in parallel with its respective physical twin. Each digital twin is linked to its respective physical twin through a unique key. The unique key is used to identify the physical twin and allows to establish a bijective (one-to-one) relationship between the digital twin and its real twin. Recently, digital twin has become the center of attention and has attracted significant attention from the Industry 4.0, research and development community, manufacturing, and others due to its importance in the improvement of the quality of products, services, processes, devices, etc. in a specific context using AI techniques.

There is a large number of industries such as manufacturing and aviation, which have been developing and commercializing the digital twin in order to optimize their processes since last years [151]. In addition, the digital twin is also in its initial stage in healthcare and medicine fields and is expected to be fully commercialized with the development and deployment of 6G communication networks in the near future [152]. Despite, it is also being extensively applied in the IoT and Industry 4.0 domains. In both scenarios, the AI techniques are used to collect, analyze, and test (in different conditions) the data from a physical object to build its softwarized copy [153]. The more information about the physical object is provided to the AI analyzer, the more accurate and better the performance and prediction of the virtual object will be during its life cycle [154].

Towards full utilization of digital twin in $6 \mathrm{G}$ communication system and specifically in the context of Industry 4.0 and IoT, there are still a number of very critical research problems, which are needed to be addressed from both academia and industry. They are, including, but not limited to: dynamically scaling up the platform of digital twin to millions and billions of IoT devices, the deployment of the zero-touch and selfmanagement approaches to the devices and processes, lack of existing models and methodologies in the area of AI to virtualize the physical object, security and privacy, and many others that are thoroughly addressed in [149], [150], and [154]. These challenges shall be, first and foremost, explored aiming to develop more suitable digital twin solutions for a wide range of IoT deployment scenarios and industrial adaptations in the coming decade.

\section{4) Intelligent Edge Computing}

Edge computing plays a significant role in increasing the performance of network services, efficient utilization of network resources (both physical and virtual), decreasing CAPEX/OPEX of a mobile operator, and lowering network complexity (both in control plane and user plane) [155]. However, the existence of a large number of end users, each with a diverse set of business and technical requirements, challenges the network operator to think on different alternatives in order to address the existing limitations related to edge computing using cutting-edge AI tools and modern ML methods. To that objective, the edge intelligence (EI) is introduced aiming to integrate the $\mathrm{AI}$ and ML techniques at the edge of the mobile networks in order to bring automation and intelligence. The
EI is envisioned to be one of the key enabling technologies for beyond $5 \mathrm{G}$ and $6 \mathrm{G}$ communication networks. Thanks to the increasing number of smart portable devices, user equipment, internet of intelligent things (IoIT), and the proliferation of intelligent services; there is a strong demand for the EI in the edge of $6 \mathrm{G}$ mobile networks to automate its respective tasks [103].

One of the major use cases of EI can be the automation of the management and orchestration tasks of the virtual resources in NG-RAN architecture. In this use case, the EI is extended to the NG-RAN in order to automate all tasks related to the RAN network slice subnet management function (NSSMF) and network function management functions (NFMFs) in order to lower the management and orchestration complexity. To that aim, the ETSI has launched the ENI ISG to investigate and provides recommendations to operators [156]. In each of the use cases, including the aforementioned, the EI is constituted of a set of connected devices which are used to collect, normalize, process, and analyze the data. Subsequently, the processed data is sent back to the assisted systems in the form of recommendations and/or orders to be executed in order to automate the target tasks or functionalities [156].

The applications of EI in the context of vertical industries have also attracted the attention of both academia and industrial organizations. For example, the EI plays significant role in addressing mission-critical applications and massive and critical mMTC types of services in centralized, semi-centralized, and localized resource allocation scenarios. Despite, the EI also provides energy-efficient solution which are expected to reduce the energy consumption of the communication networks. Therefore, it is considered a novel opportunity for both operators and vertical industries in order to digitize its application and businesses - using personal computing, fog computing, urban computing, and other mechanisms - in the $6 \mathrm{G}$ mobile networks.

Despite the aforementioned key advantages, there are still a number of unsolved research challenges in realising EI in beyond $5 \mathrm{G}$ mobile networks. It is, therefore, crucial to identify and analyze such open research problems and seek for their theoretical and technical solutions. Among others, the most prominent challenges are: data consistency on every device at edge, data scarcity at edge, bad adaptability of statically trained model, and data privacy and security. We expect that more research efforts are needed to completely realize EI in beyond $5 \mathrm{G}$ and $6 \mathrm{G}$ networks upon addressing such challenges.

5) Communication-Computing-Control Convergence

Mobile edge networks provide computing and caching capabilities at the network edge, which makes low-latency, high-bandwidth, location-aware pervasive computing services a reality. With the proliferation of IoT and Tactile Internet, a huge number of sensing devices and actuators are connected to mobile networks. The next-generation system is envisioned to become a huge computer that would converge ubiquitous communication, computation, storage, sensing, and controlling as a whole to provide disruptive applications.

Due to its superiority in integration and mobility over wired connections, wireless links are gradually relied by modern and 
future controlling systems to close the signal loop, giving birth to the use scenario of URLLC. The spirit of system design behind this concept follows the classic methodology of control \& communication independent design: it starts from designing the controlling component without concerning the characteristics of communication system, where a set of communication requirements will be generated regarding the expected controlling performance; then there follows the designing of wireless system, aiming at achieving the target performance proposed by the last stage. The KPI requirements of URLLC, such as $99.999 \%$ reliability and $10 \mathrm{~ms}$, were formulated for a generic controlling scenario in this fashion. Nevertheless, recent studies have revealed a necessity of inloop co-designing of communication and controlling systems tightly coupled to each other. For instance, the close-loop reliability of controlling system has been proven exponentially decreasing along with the AoI over the feedback channel [157], [158]. Meanwhile, the AoI over control/feedback channels, as a communication metric, is convex about the arrival rates of controlling command and feedback information, which correspond to the sampling rate of sensors and decision rate of controller in the control system [159]. The performance of communication system, is therefore limited by the design of sensing and controlling systems.

Similar issues are also to be addressed in cloud computing. While it occasionally happens that some computing task occupies the cloud server for long time, blocking all other pending tasks in the waiting queue and causing a severe congestion, its source may have re-issued the same task with a more up-to-date status, making the previous task outdated and lack of utility. A preemption of the server, i.e. terminating the ongoing task in advance to its completion, will in this case help reduce the age of task and improve the quality of cloud computing service [160]. Furthermore, it is also critical for reducing the AoI, to schedule the order of computing tasks from multiple applications to be offloaded to the cloud [46]. Optimal decisions of such preemption and scheduling, however, cannot be solely solved by the computing server, nor by the communication system, but only achievable in a joint collaboration among the terminal devices, the network controller, and the cloud computing server.

With the dramatically increasing demand for wirelessconnected industrial automation, and the irreversible trend that not only most mobile applications but also the mobile networking service itself are gradually cloudified, new approaches are therefore required in $6 \mathrm{G}$ to jointly design and optimize this communication-computing-control symbiosis [161].

\section{CONCLUSIONS}

This article provided a comprehensive survey on the drivers, requirements, efforts, and enablers for the next-generation mobile system beyond $5 \mathrm{G}$. It can be concluded that the traditional evolution of a new generation every decade will not terminate at $5 \mathrm{G}$ and the first $6 \mathrm{G}$ network is expected to be deployed in 2030 or even earlier taking into account passions of developing $6 \mathrm{G}$ from both academia and industry. $6 \mathrm{G}$ will accommodate the use cases and applications introduced in $5 \mathrm{G}$ such as IoT, Industry 4.0, virtual reality, and automatic driving with better quality of experience in a more cost-efficient, energy-efficient, and resource-efficient manner. Meanwhile, it will enable unprecedented use cases that cannot be supported by $5 \mathrm{G}$, e.g., holographic-type communications, pervasive intelligence, and global ubiquitous connectability, as well as other disruptive applications that we are unable to yet imagine. The trend of mobile communication services expanded from only human centric to connecting also machines and things, started when MTC and IoT were introduced in the age of $5 \mathrm{G}$ will continue, and IoE will be realized when $6 \mathrm{G}$ comes. The $6 \mathrm{G}$ system will meet extremely stringent requirements on latency, reliability, mobility, and security, as well as provisioning a substantial boost of coverage, peak data rate, user experienced rate, system capacity, and connectivity density, gaining KPIs generally 10 to 100 times better in comparison with 5G.

It is envisioned that $6 \mathrm{G}$ will take unprecedented transformations that will make it dramatically distinguishing with the previous generations. That is:

- It will be shifted from a radio communication network based on electronic technologies to a radio-optical system taking advantage of both electronic and photonic technologies so as to exploit the abundant spectral resources in Terahertz and visible light bands, especially in indoor optical wireless coverage, to meet the inexorable demand on higher system capacity and peak data rates.

- It will become an intelligent platform that deeply combining AI and mobile networks, where AI-as-a-Service is provided to end users through pervasive intelligence, while AI-empowered methodologies are applied to implement intelligent optimization and management of resources and networks.

- With the disruptive advances in large-scale LEO satellite constellation, the $6 \mathrm{G}$ system will go beyond terrestrial networks and provide ubiquitous coverage of the whole surface of our planet through an integrated space-aerialterrestrial network.

- It will be a smart compute-connect platform by means of converging distributed communication, computing, storage, and big data resources, integrating sensing, localization, and controlling capabilities, and inter-working with AI, blockchain, digital twin, etc.

- It should be an intelligent, green, sustainable, and secure system to fully support the informationized and intelligentized society of 2030 and beyond.

In 1926, engineer and inventor Nikola Tesla stated that "When wireless is perfectly applied the whole Earth will be converted into a huge brain". This prophecy will transform into a reality when $6 \mathrm{G}$ comes.

\section{GLOSSARY}

1G first generation

2D two-dimensional

3D three-dimensional

3G third generation

3GPP third generation partnership project

4G fourth generation 
5G fifth generation

5G-PPP fifth generation private public partnership

6G sixth generation

AI artificial intelligence

AoI age of information

AR augmented reality

AoS age of synchronization

ATIS Alliance for Telecommunications Industry Solutions

CAPEX capital expenditure

CDMA code division multiple access

CoMP coordinated multi-point

CR cognitive radio

CU centralized unit

DARPA Defense Advanced Research Projects Agency

DSM dynamic spectrum management

DU distributed unit

E2E end-to-end

EI edge intelligence

eMBB enhanced mobile broad-band

ENI experiential network intelligence

ER extended reality

ETSI European Telecommunication Standard Institute

FCC Federal Communications Commission

FSO free-space optical

GEO geostationary Earth orbit

gNB next-generation NodeB

HAP high-altitude platform

HTC holographic-type communication

IAB integrated access and backhaul

ICT information and communication technology

IDMA interleave division multiple access

IMT International Mobile Telecommunications

IoE internet of everything

IoIT internet of intelligent things

IoT internet of things

IR infrared

IRS intelligent reflecting surfaces

ISG industry specification group

ISTN integrated space and terrestrial network

ITU-R International Telecommunciation Union - Radiocommunication

ITU-T International Telecommunciation Union - Telecommunication

KPI key performance indicator

LBT listen-before-talk

LD Laser diode

LED light-emitting diodes

LEO low Earth orbit

LOS line of sight

LTE long term evolution

M2M machine to machine

MBB mobile broadband

MIMO multi-input multi-output

ML machine learning

mMTC massive machine-type communications

mmWave millimeter wave

mULC massive ultra-reliable low-latency communication

MUSA multi-user shared access
NFMF network function management function

NFV network function virtualization

MANO management and orchestration

NG-RAN next-generation radio access network

NOMA non-orthogonal multiple access

NR new radio

NSSMF network slice subnet management function

OFDM orthogonal frequency-division multipling

OMA orthogonal multiple access

OPEX operational expenditure

O-RAN open radio access network

PD power domain

PDMA pattern-division multiple access

PNF physical network function

PoP point of presence

PPP public-private partnership

PRB physical resource block

QoS quality of service

RAN radio access network

RAT radio access technology

$\mathbf{R F}$ radio frequency

RIS reconfigurable intelligent surfaces

RU radio unit

SCMA sparse-code multiple access

SDN software-defined networking

SLAM simultaneous localization and mapping

SLM spatial light modulator

SRE smart radio environment

SWIPT simultaneous wireless information and power transfer

TCMA trellis-coded multiple access

THz Terahertz

UAV unmanned aerial vehicle

ULBC ultra-reliable low-latency broadband communication

UMBB ubiquitous mobile broadband

umMTC ultra-massive machine-type communication

URLLC ultra-reliable low-latency communications

VLC visible light communication

VNF virtual network function

VR virtual reality

WSN wireless sensor network

\section{REFERENCES}

[1] J. D. Vriendt et al., "Mobile network evolution: a revolution on the move," IEEE Commun. Mag., vol. 40, no. 4, pp. 104-111, Apr. 2002.

[2] E. Dahlman et al., "UMTS/IMT-2000 based on wideband CDMA," IEEE Commun. Mag., vol. 36, no. 9, pp. 70-80, Sep. 1998.

[3] D. Astely et al., "LTE: the evolution of mobile broadband," IEEE Commun. Mag., vol. 47, no. 4, pp. 44-51, Apr. 2009.

[4] J. G. Andrews et al., "What will 5G be?" IEEE J. Sel. Areas Commun., vol. 32, no. 6, pp. 1065-1082, Jun. 2014

[5] F. H. P. Fitzek and P. Seeling, "Why we should not talk about 6G," arXiv, Mar. 2020.

[6] "A blueprint of technology, applications and market drivers towards the year 2030 and beyond," White Paper, ITU-T FG-NET-2030, May 2019.

[7] "Key drivers and research challenges for $6 \mathrm{G}$ ubiquitous wireless intelligence,” White Paper, 6G Flagship, University of Oulu, Sep. 2019.

[8] K. David and H. Berndt, "6G vision and requirements: Is there any need for beyond 5G?" IEEE Veh. Technol. Mag., vol. 13, no. 3, pp. 72-80, Sep. 2018

[9] S. J. Nawaz et al., "Quantum machine learning for $6 \mathrm{G}$ communication networks: State-of-the-art and vision for the future," IEEE Access, vol. 7, pp. 46317-46350, Apr. 2019. 
[10] T. S. Rappaport et al., "Wireless communications and applications above $100 \mathrm{GHz}$ : Opportunities and challenges for 6G and beyond," IEEE Access, vol. 7, pp. 78 729-78 757, Jun. 2019.

[11] P. Yang et al., "6G wireless communications: Vision and potential techniques," IEEE Netw., vol. 33, no. 4, pp. 70-75, Jul. 2019.

[12] K. B. Letaief et al., "The roadmap to 6G: AI empowered wireless networks," IEEE Commun. Mag., vol. 57, no. 8, pp. 84-90, Aug. 2019.

[13] B. Zong et al., "6G technologies: Key drivers, core requirements, system architectures, and enabling technologies," IEEE Veh. Technol. Mag., vol. 14, no. 3, pp. 18-27, Sep. 2019.

[14] Z. Zhang et al., "6G wireless networks: Vision, requirements, architecture, and key technologies," IEEE Veh. Technol. Mag., vol. 14, no. 3 , pp. 28-41, 2019.

[15] E. C. Strinati et al., "6G: The next frontier: From holographic messaging to artificial intelligence using subterahertz and visible light communication,” IEEE Veh. Technol. Mag., vol. 14, no. 3, pp. 42-50, Sep. 2019.

[16] T. Huang et al., "A survey on green 6G network: Architecture and technologies," IEEE Access, vol. 7, pp. 175 758-175 768, Dec. 2019.

[17] W. Jiang and F.-L. Luo, "Computational radio intelligence: One key for 6G wireless," ZTE Commun., vol. 17, no. 4, pp. 1-3, Dec. 2019

[18] S. Dang et al., "What should 6G be?" Nat. Electron., vol. 3, p. 20-29, Jan. 2020.

[19] F. Tang et al., "Future intelligent and secure vehicular network toward 6G: Machine-learning approaches," Proc. IEEE, vol. 108, no. 2, pp. 292-307, Feb. 2020

[20] M. Giordani et al., "Toward 6G networks: Use cases and technologies," IEEE Commun. Mag., vol. 58, no. 3, pp. 55-61, Mar. 2020.

[21] H. Viswanathan and P. E. Mogensen, "Communications in the 6G era," IEEE Access, vol. 8, pp. $57063-57$ 074, Mar. 2020.

[22] S. Zhang, C. Xiang, and S. XU, "6G: Connecting everything by 1000 times price reduction," IEEE Open J. of Veh. Techno., vol. 1, pp. 107115, Mar. 2020.

[23] S. Chen et al., "Vision, requirements, and technology trend of 6G: How to tackle the challenges of system coverage, capacity, user data-rate and movement speed," IEEE Wireless Commun. Mag., vol. 27, no. 2, pp. 218-228, Apr. 2020

[24] W. Saad et al., "A vision of 6G wireless systems: Applications, trends, technologies, and open research problems," IEEE Netw., vol. 34, no. 3, pp. 134-142, May 2020.

[25] N. Kato et al., "Ten challenges in advancing machine learning technologies toward 6G," IEEE Wireless Commun. Mag., vol. 27, no. 3, pp. 96-103, Jun. 2020

[26] W. Guo, "Explainable artificial intelligence for 6G: Improving trust between human and machine," IEEE Commun. Mag., vol. 58, no. 6 , pp. 39-45, Jun. 2020.

[27] M. Z. Chowdhury et al., "6G wireless communication systems: Applications, requirements, technologies, challenges, and research directions," IEEE Open Journal of the Communications Society, vol. 1, pp. 957-975, Jul. 2020.

[28] F. Tariq et al., "A speculative study on 6G," IEEE Wireless Commun. Mag., vol. 27, no. 4, pp. 118-125, Aug. 2020.

[29] G. Gui et al., "6G: Opening new horizons for integration of comfort, security, and intelligence," IEEE Wireless Commun. Mag., vol. 27, no. 5, pp. 126-132, Oct. 2020.

[30] C. Huang et al., "Holographic MIMO surfaces for $6 \mathrm{G}$ wireless networks: Opportunities, challenges, and trends," IEEE Wireless Commun. Mag., vol. 27, no. 5, pp. 118-125, Oct. 2020.

[31] L. Bariah et al., "A prospective look: Key enabling technologies, applications and open research topics in 6G networks," IEEE Access, vol. 8, pp. 174 792-174820, Oct. 2020.

[32] IMT traffic estimates for the years 2020 to 2030, ITU-R Std. M.2370-0, Jul. 2015.

[33] "Mobile data traffic outlook," Report, Ericsson, Jun. 2020

[34] Microsoft HoloLens. [Online]. Available: https://www.microsoft.com/ en-us/hololens/

[35] A. Clemm et al., "Toward truly immersive holographic-type communication: Challenges and solutions," IEEE Commun. Mag., vol. 58, no. 1, pp. 93-99, Jan. 2020.

[36] "Cloud VR network solution white paper," White Paper, HUAWEI, 2018.

[37] G. Fettweis et al., "The Tactile Internet," Technology Watch Report, ITU-T, Aug. 2014.

[38] G. Fettweis, "The tactile internet: Applications and challenges," IEEE Veh. Technol. Mag., vol. 9, no. 1, pp. 64-70, Mar. 2014

[39] Boston dynamics. [Online]. Available: https://www.bostondynamics. com/atlas
[40] Spacex Starlink. [Online]. Available: https://www.starlink.com/

[41] Z. Qu et al., "LEO satellite constellation for internet of things," IEEE Access, vol. 5, pp. 18391-18 401, Sep. 2017.

[42] Y. Hu and V. O. K. Li, "Satellite-based internet: A tutorial," IEEE Commun. Mag., vol. 39, no. 3, pp. 154-162, Mar. 2001.

[43] IMT vision-Framework and overall objectives of the future development of IMT for 2020 and beyond, ITU-R Std. M.2083-0, Sep. 2015.

[44] Minimum requirements related to technical performance for IMT-2020 radio interface(s), ITU-R Std. M.2410-0, Nov. 2017.

[45] A. Kosta, N. Pappas, and V. Angelakis, Age of Information: A New Concept, Metric, and Tool, 2017.

[46] X. Song et al., "Age based task scheduling and computation offloading in mobile-edge computing systems," in Proc. IEEE Wireless Commun Netw. Conf. Workshops (WCNCW, 2019, pp. 1-6.

[47] H. Tang et al., "Scheduling to minimize age of synchronization in wireless broadcast networks with random updates," IEEE Trans. Wireless Commun., vol. 19, no. 6, pp. 4023-4037, 2020.

[48] A. Ghosh et al., "5G evolution: A view on 5G cellular technology beyond 3GPP release 15," IEEE Access, vol. 7, pp. 127639-127651, Sep. 2019.

[49] 5g-complete. [Online]. Available: https://5gcomplete.eu/

[50] 5g-clarity. [Online]. Available: https://www.5gclarity.com/

[51] Netx G Alliance. [Online]. Available: https://nextgalliance.org/

[52] X. Wang et al., "Millimeter wave communication: A comprehensive survey," IEEE Commun. Surveys Tuts., vol. 20, no. 3, pp. 1616-1653, 2018.

[53] L. J. Ippolito, "Radio propagation for space communications systems," Proc. IEEE, vol. 69, no. 6, pp. 697-727, 1981

[54] H. Ajorloo and M. T. Manzuri-Shalmani, "Modeling beacon period length of the uwb and 60-ghz mmwave wpans based on ecma-368 and ecma-387 standards," IEEE Trans. Mobile Comput., vol. 12, no. 6, pp. 1201-1213, 2013.

[55] "IEEE 802.15 WPAN Millimeter Wave Alternative PHY Task Group 3c (TG3c)," Available: http://www.ieee802. org/15/pub/TG3c.html.

[56] T. Nitsche et al., "Ieee 802.11ad: directional $60 \mathrm{ghz}$ communication for multi-gigabit-per-second wi-fi [invited paper]," IEEE Commun. Mag., vol. 52, no. 12, pp. 132-141, 2014.

[57] B. Han, L. Wang, and H. D. Schotten, "A 3D human body blockage model for outdoor millimeter-wave cellular communication," Phys. Commun., vol. 25, pp. 502-510, 2017.

[58] A. M. Al-samman, M. H. Azmi, and T. Abd Rahman, "A survey of millimeter wave (mm-Wave) communications for 5G: Channel measurement below and above $6 \mathrm{GHz}$," in International Conference of Reliable Information and Communication Technology. Springer, 2018, pp. 451-463.

[59] Z. Chen et al., "A survey on terahertz communications," China Commun., vol. 16, no. 2, pp. 1-35, 2019.

[60] B. Han, M. A. Habibi, and H. D. Schotten, "Optimal resource dedication in grouped random access for massive machine-type communications," in $n$ Proc. IEEE Conf. Standards Commun. Netw. (CSCN), 2017, pp. 72-77

[61] H. Vettikalladi et al., "Sub-thz antenna for high-speed wireless communication systems," Int. J. Antennas Propag., vol. 2019, 2019.

[62] J. C. Tucek et al., "Operation of a compact 1.03 thz power amplifier," in Proc. IEEE Int. Vac. Electron. Conf. (IVEC). IEEE, 2016, pp. 1-2.

[63] S. Koenig et al., "Wireless sub-thz communication system with high data rate," Nat. Photonics, vol. 7, no. 12, pp. 977-981, 2013.

[64] A. Sevincer et al., "Lightnets: Smart lighting and mobile optical wireless networks - a survey," IEEE Commun. Surveys Tuts., vol. 15 , no. 4, pp. $1620-1641,2013$

[65] A. Al-Kinani et al., "Optical wireless communication channel measurements and models," IEEE Commun. Surveys Tuts., vol. 20, no. 3, pp. 1939-1962, 2018.

[66] P. H. Pathak et al., "Visible light communication, networking, and sensing: A survey, potential and challenges," IEEE Commun. Surveys Tuts., vol. 17, no. 4, pp. 2047-2077, 2015.

[67] L. Zeng et al., "High data rate multiple input multiple output (mimo) optical wireless communications using white led lighting," IEEE J. Sel. Areas Commun., vol. 27, no. 9, pp. 1654-1662, 2009.

[68] A. Nuwanpriya, S. Ho, and C. S. Chen, "Indoor mimo visible light communications: Novel angle diversity receivers for mobile users," IEEE J. Sel. Areas Commun., vol. 33, no. 9, pp. 1780-1792, 2015.

[69] N. Huang, X. Wang, and M. Chen, "Transceiver design for mimo vlc systems with integer-forcing receivers," IEEE J. Sel. Areas Commun., vol. 36 , no. 1 , pp. 66-77, 2018. 
[70] L. Wu, Z. Zhang, and H. Liu, "Transmit beamforming for mimo optical wireless communication systems," Wirel. Pers. Commun., vol. 78, no. 1, pp. 615-628, 2014.

[71] Y. Zhuang et al., "A survey of positioning systems using visible led lights," IEEE Commun. Surveys Tuts., vol. 20, no. 3, pp. 1963-1988, 2018.

[72] A. Memedi and F. Dressler, "Vehicular visible light communications: A survey," IEEE Commun. Surveys Tuts., pp. 1-1, 2020.

[73] M. A. Arfaoui et al., "Physical layer security for visible light communication systems: A survey," IEEE Commun. Surveys Tuts., vol. 22, no. 3, pp. 1887-1908, 2020.

[74] H. Elgala et al., "Indoor optical wireless communication: potential and state-of-the-art," IEEE Commun. Mag., vol. 49, no. 9, pp. 56-62, Sep. 2011.

[75] J. M. Kahn and J. R. Barry, "Wireless infrared communications," Proc. IEEE, vol. 85, no. 2, pp. 265-298, Feb. 1997.

[76] T. Ohtsuki, "Multi-subcarrier modulation in optical wireless communication," IEEE Commun. Mag., vol. 41, no. 3, pp. 74-79, Mar. 2003.

[77] L. Zeng et al., "High data rate multiple input multiple output (MIMO) optical wireless communications using white LED lighting," IEEE $J$. Sel. Areas Commun., vol. 27, no. 9, pp. 1654-1662, Dec. 2009.

[78] J. C. Juarez et al., "Free-space optical communications for nextgeneration military networks," IEEE Commun. Mag., vol. 44, no. 11, pp. 46-51, Nov. 2006.

[79] Z. Xu and B. M. Sadler, "Ultraviolet communications: Potential and state-of-the-art," IEEE Commun. Mag., vol. 46, no. 5, pp. 67-73, May 2008

[80] M. Marcus, J. Burtle, B. Franca, A. Lahjouji, and N. McNeil, "Federal communications commission spectrum policy task force," Report of the Unlicensed Devices and Experimental Licenses Working Group, 2002.

[81] S. Haykin, "Cognitive radio: brain-empowered wireless communications," IEEE J. Sel. Areas Commun., vol. 23, no. 2, pp. 201-220, 2005.

[82] A. M. Voicu, L. Simić, and M. Petrova, "Inter-technology coexistence in a spectrum commons: A case study of Wi-Fi and LTE in the 5-GHz unlicensed band," IEEE J. Sel. Areas Commun., vol. 34, no. 11, pp. 3062-3077, 2016.

[83] I. F. Akyildiz, A. Kak, and S. Nie, " $6 \mathrm{G}$ and beyond: The future of wireless communications systems," IEEE Access, vol. 8, pp. 133995 134030,2020

[84] A. Kliks et al., "Beyond 5G: Big data processing for better spectrum utilization," IEEE Veh. Technol. Mag., vol. 15, no. 3, pp. 40-50, 2020.

[85] M. A. Habibi et al., "A comprehensive survey of ran architectures toward 5g mobile communication system," IEEE Access, vol. 7, pp. 70 371-70 421, 2019.

[86] A. Laghrissi and T. Taleb, "A survey on the placement of virtual resources and virtual network functions," IEEE Commun. Surveys Tuts., vol. 21, no. 2, pp. 1409-1434, 2019.

[87] W. Jiang, M. Strufe, and H. D. Schotten, "Experimental results for artificial intelligence-based self-organized 5G networks," in Proc. IEEE Int. Symp. on Personal, Indoor and Mobile Radio Commun. (PIMRC), Montreal, QC, Canada, Oct. 2017.

[88] W. Jiang, M. Strufe, and H. Schotten, "Intelligent network management for 5G systems: The SELFNET approach," in Proc. IEEE Eur. Conf. on Net. and Commun. (EUCNC), Oulu, Finland, Jun. 2017, pp. 109-113.

[89] Y. Wang et al., "Network management and orchestration using artificial intelligence: Overview of etsi eni," IEEE Commun. Standards Mag., vol. 2, no. 4, pp. 58-65, 2018.

[90] A. Ksentini, M. Bagaa, and T. Taleb, "On using SDN in 5G: The controller placement problem," in Proc. IEEE GLOBECOM, 2016, pp. $1-6$.

[91] V.-M. Alevizaki et al., "Joint fronthaul optimization and SDN controller placement in dynamic 5G networks," in Optical Network Design and Modeling. Cham: Springer International Publishing, 2020, pp. 181192.

[92] S. Khan et al., "Topology discovery in software defined networks: Threats, taxonomy, and state-of-the-art," IEEE Commun. Surveys Tuts., vol. 19, no. 1, pp. 303-324, 2017.

[93] W. Jiang, M. Strufe, and H. D. Schotten, "Autonomic network management for software-define and virtualized 5G systems," in Proc. Eur. Wireless, Dresden, Germany, May 2017.

[94] P. Chemouil et al., "Special issue on artificial intelligence and machine learning for networking and communications," IEEE J. Sel. Areas Commun., vol. 37, no. 6, pp. 1185-1191, 2019

[95] S. Tomovic and I. Radusinovic, "Toward a scalable, robust, and QoSaware virtual-link provisioning in SDN-based ISP networks," IEEE Trans. Netw. Service Manag., vol. 16, no. 3, pp. 1032-1045, 2019.
[96] S. E. Elayoubi et al., "5g ran slicing for verticals: Enablers and challenges," IEEE Commun. Mag., vol. 57, no. 1, pp. 28-34, 2019.

[97] M. A. Habibi, B. Han, and H. D. Schotten, "Network Slicing in 5G Mobile Communication: Architecture, Profit Modeling, and Challenges," in Proceedings of the 14th International Symposium on Wireless Communication Systems - 2017, Sep. 28 - Oct. 1, 2017, Bologna, Italy, accessed: 20-Sep.-2020.

[98] “3GPP TS 28.801, Technical Specification Group Services and System Aspects; Telecommunication management; Study on management and orchestration of network slicing for next generation network (Release 15). V15 1.0," january 2018.

[99] "ETSI GR NFV-EVE 012 V3.1.1, Network Functions Virtualisation (NFV) Release 3; Evolution and Ecosystems; Report on Network Slicing Support with ETSI NFV Architecture Framework," december 2017.

[100] H. Hirayama et al., "Ran slicing in multi-cu/du architecture for $5 \mathrm{~g}$ services," in Proc. IEEE Vehicular Tech. Conf. (VTC), 2019, pp. 1-5.

[101] "ETSI GS NFV-INF 001 V1.1.1, Network Functions Virtualisation (NFV); Infrastructure Overview," january 2015.

[102] M. A. Habibi et al., "How should network slice instances be provided to multiple use cases of a single vertical industry?" IEEE Commun. Standards Mag., vol. 4, no. 3, pp. 53-61, 2020.

[103] M. A. Habibi et al., "Towards a Fully Virtualized, Cloudified, and Slicing-aware RAN for 6G Mobile Networks."

[104] _ "The Structure of Service Level Agreement of Slice-based 5G Network," 2018, in Proceedings of the 29th International Symposium on Personal, Indoor and Mobile Radio Communications - 2018, Sep. 09 - Sep. 12, 2018, Bologna, Italy, accessed: 20-Sep.-2020.

[105] O.-R. Alliance, "O-RAN: Towards an Open and Smart RAN," published: October 1, 2018, Accessed: September 26, 2020.

[106] - "O-RAN Use Cases and Deployment Scenarios: Towards Open and Smart RAN," published: February 1, 2020, Accessed: September 26, 2020.

[107] E. Björnson et al., "Massive mimo is a reality—what is next?: Five promising research directions for antenna arrays," Digit. Signal Process., vol. 94, pp. 3-20, 2019.

[108] C. Huang et al., "Holographic mimo surfaces for $6 \mathrm{~g}$ wireless networks: Opportunities, challenges, and trends," IEEE Wireless Commun. Mag., pp. $1-8,2020$.

[109] M. A. El Mossallamy et al., "Reconfigurable intelligent surfaces for wireless communications: Principles, challenges, and opportunities," IEEE Trans. Cogn. Commun. Netw., pp. 1-1, 2020.

[110] M. D. Renzo et al., "Smart radio environments empowered by reconfigurable intelligent surfaces: How it works, state of research, and road ahead," IEEE J. Sel. Areas Commun., pp. 1-1, 2020.

[111] G. T. 36.819, "Coordinated multi-point operation for lte physical layer aspects," 2013

[112] Q. C. Li et al., "5g network capacity: Key elements and technologies," IEEE Veh. Technol. Mag., vol. 9, no. 1, pp. 71-78, 2014.

[113] H. Q. Ngo et al., "Cell-free massive mimo versus small cells," IEEE Trans. Wireless Commun., vol. 16, no. 3, pp. 1834-1850, 2017.

[114] E. Björnson and L. Sanguinetti, "Cell-free versus cellular massive mimo: What processing is needed for cell-free to win?" in Proc. IEEE 20th Int. Workshop Signal Process. Adv. Wirel. Commun. (SPAWC), 2019, pp. 1-5.

[115] S. Bassoy et al., "Coordinated multi-point clustering schemes: A survey," IEEE Commun. Surveys Tuts., vol. 19, no. 2, pp. 743-764, 2017.

[116] V. Kotzsch and G. Fettweis, "Interference analysis in time and frequency asynchronous network MIMO OFDM systems," in 2010 IEEE Wireless Communication and Networking Conference, 2010, pp. 1-6.

[117] W. Jiang and T. Kaiser, "From OFDM to FBMC: Principles and Comparisons," in Signal Processing for 5G: Algorithms and Implementations, F. L. Luo and C. Zhang, Eds. United Kindom: John Wiley\&Sons and IEEE Press, 2016, ch. 3.

[118] S. M. R. Islam et al., "Power-domain non-orthogonal multiple access (noma) in 5g systems: Potentials and challenges," IEEE Commun Surveys Tuts., vol. 19, no. 2, pp. 721-742, 2017.

[119] T. Aulin and R. Espineira, "Trellis coded multiple access (TCMA)," in Proc. IEEE Int. Conf. on Commun.ICC, vol. 2, 1999, pp. 1177-1181 vol.2.

[120] Li Ping et al., "Interleave division multiple-access," IEEE Trans. Wireless Commun., vol. 5, no. 4, pp. 938-947, 2006.

[121] Z. Yuan et al., "Multi-user shared access for internet of things," in Proc. IEEE Vehicular Tech. Conf. (VTC), 2016, pp. 1-5. 
[122] S. Chen et al., "Pattern division multiple access-a novel nonorthogonal multiple access for fifth-generation radio networks," IEEE Trans. Veh. Technol., vol. 66, no. 4, pp. 3185-3196, 2017.

[123] H. Nikopour and H. Baligh, "Sparse code multiple access," in Proc. IEEE Int. Symp. on Personal, Indoor and Mobile Radio Commun. (PIMRC), 2013, pp. 332-336.

[124] J. Grubb, “The traveler's dream come true (satellite personal communication)," IEEE Commun. Mag., vol. 29, no. 11, pp. 48-51, Nov. 1991.

[125] J. Foust, "SpaceX's space-internet woes: Despite technical glitches, the company plans to launch the first of nearly 12,000 satellites in 2019 ," IEEE Spectrum, vol. 56, no. 1, pp. 50-51, Jan. 2019.

[126] S. Karapantazis and F.-N. Pavlidou, "The role of high altitude platforms in beyond 3G networks," IEEE Commun. Mag., vol. 12, no. 6, pp. 3341, Dec. 2005.

[127] W. Jiang et al., "Achieving high reliability in aerial-terrestrial networks: Opportunistic space-time coding," in Proc. IEEE Eur. Conf. on Net. and Commun. (EUCNC), Bologne, Italy, Jun. 2014.

[128] A. Mohammed et al., "The role of high-altitude platforms (HAPs) in the global wireless connectivity," Proc. IEEE, vol. 99, no. 11, pp. 19391953, Nov. 2011

[129] W. Jiang et al., "Opportunistic relaying over aerial-to-terrestrial and device-to-device radio channels," in Proc. IEEE Intl. Conf. on Commun.ICC, Sydney, Australia, Jul. 2014, pp. 206-211.

[130] F. Dovis et al., "Small-scale fading for high-altitude platform (HAP) propagation channels," IEEE J. Sel. Areas Commun., vol. 20, no. 3 , pp. 641-647, Apr. 2002.

[131] E. T. Michailidis and A. G. Kanatas, "Three-dimensional HAP-MIMO channels: Modeling and analysis of space-time correlation," IEEE Trans. Veh. Technol., vol. 59, no. 5, pp. 2232-2242, Jun. 2010.

[132] F. Fidler et al., "Optical communications for high-altitude platforms," IEEE J. Sel. Topics Quantum Electron., vol. 16, no. 5, pp. 1058-1070, Sep. 2010.

[133] A. Fotouhi et al., "Survey on uav cellular communications: Practical aspects, standardization advancements, regulation, and security challenges," IEEE Commun. Surveys Tuts., vol. 21, no. 4, pp. 3417-3442, 2019.

[134] H. Wang et al., "Deployment algorithms of flying base stations: $5 \mathrm{~g}$ and beyond with uavs," IEEE Internet Things J., vol. 6, no. 6, pp. 10 009-10 027, 2019.

[135] C. Su et al., "Uav-assisted wireless charging for energy-constrained iot devices using dynamic matching," IEEE Internet Things J., vol. 7, no. 6, pp. 4789-4800, 2020 .

[136] W. Feng et al., "Uav-enabled swipt in iot networks for emergency communications," IEEE Trans. Wireless Commun., vol. 27, no. 5, pp. 140-147, 2020.

[137] W. Jiang and H. D. Schotten, "A deep learning method to predict fading channel in multi-antenna systems," in Proc. IEEE Veh. Tech. Conf. (VTC), Antwerp, Belgium, May 2020.

[138] _ "Multi-antenna fading channel prediction empowered by artificial intelligence," in Proc. IEEE Veh. Tech. Conf. (VTC), Chicago, USA, Aug. 2018.

[139] W. Jiang, M. Strufe, and H. Schotten, "A SON decision-making framework for intelligent management in 5G mobile networks," in Proc. IEEE Intl. Conf. on Compu. and Commun. (ICCC), Chengdu, Chia, Dec. 2017.

[140] W. Jiang and H. D. Schotten, "Deep learning for fading channel prediction," IEEE Open J. of the Commun. Society, vol. 1, pp. 320-332, Mar. 2020

[141] H. Huang et al., "Deep-learning-based millimeter-wave massive MIMO for hybrid precoding," IEEE Trans. Veh. Technol., vol. 68, no. 3, pp. 3027-3032, Mar. 2019.

[142] W. Jiang and H. D. Schotten, "Neural network-based fading channel prediction: A comprehensive overview," IEEE Access, vol. 7, pp. $118112-118124$, Aug. 2019.

[143] — , "Recurrent neural networks with long short-term memory for fading channel prediction," in Proc. IEEE Veh. Tech. Conf. (VTC), Antwerp, Belgium, May 2020.

[144] F. Tschorsch and B. Scheuermann, "Bitcoin and beyond: A technical survey on decentralized digital currencies," IEEE Commun. Surveys Tuts., vol. 18, no. 3, pp. 2084-2123, Mar. 2016.

[145] H.-N. Dai et al., "Blockchain for internet of things: A survey," IEEE Internet Things J., vol. 6, no. 5, pp. 8076-8094, Oct. 2019.

[146] J. Xie et al., "A survey on the scalability of blockchain systems," IEEE Netw., vol. 33, no. 5, pp. 166-173, Sep. 2019.

[147] D. C.Nguyen et al., "Blockchain for 5G and beyond networks: A state of the art survey," J. Netw. Comput. Appl., vol. 166, pp. 1-38, Sep. 2020 .
[148] Z. Xiong et al., "When mobile Blockchain meets edge computing," IEEE Commun. Mag., vol. 56, no. 8, pp. 33-39, Aug. 2018.

[149] R. Minerva, G. M. Lee, and N. Crespi, "Digital twin in the iot context: A survey on technical features, scenarios, and architectural models," Proc. IEEE, vol. 108, no. 10, pp. 1785-1824, 2020.

[150] F. Pires et al., "Digital twin in industry 4.0: Technologies, applications and challenges," in Proc. IEEE Int. Conf. Ind. Informat. (INDIN), vol. 1, 2019, pp. 721-726.

[151] B. Schleich, N. Anwer, L. Mathieu, and S. Wartzack, "Shaping the digital twin for design and production engineering," CIRP Annals, vol. 66, no. 1, pp. $141-144,2017$.

[152] A. J. Zakrajsek and S. Mal, "The development and use of a digital twin model for tire touchdown health monitoring," 2017, in Proceedings of the Proc. AIAA.

[153] S. Luscinski, "Digital twinning for smart industry." EAI, 122018.

[154] B. R. Barricelli, E. Casiraghi, and D. Fogli, "A survey on digital twin: Definitions, characteristics, applications, and design implications," IEEE Access, vol. 7, pp. 167 653-167 671, 2019.

[155] M. Nasimi et al., "Edge-assisted congestion control mechanism for $5 \mathrm{~g}$ network using software-defined networking," in Proc. IEEE Int. Symp. Wireless Commun. Syst. (ISWCS), 2018, pp. 1-5.

[156] "ETSI GS ENI 005 V1.1.1, Experiential Networked Intelligence (ENI); System Architecture," september 2019.

[157] O. Ayan, M. Vilgelm, and W. Kellerer, "Optimal scheduling for discounted age penalty minimization in multi-loop networked control," in Proc. IEEE Consum. Commun. Netw. Conf. (CCNC), 2020, pp. 1-7.

[158] B. Han et al., "Robustness analysis of networked control systems with aging status," in Proc. 2020 IEEE Conf. Computer Commun. Wksps., 2020, pp. 1360-1361.

[159] Z. Jiang et al., "Ai-assisted low information latency wireless networking," IEEE Wireless Commun. Mag., vol. 27, no. 1, pp. 108-115, 2020.

[160] A. Arafa, R. D. Yates, and H. V. Poor, "Timely cloud computing: Preemption and waiting," in 2019 57th Annual Allerton Conference on Communication, Control, and Computing (Allerton), 2019, pp. 528535.

[161] G. Zhao et al., "Toward real-time control in future wireless networks: Communication-control co-design," IEEE Commun. Mag., vol. 57, no. 2, pp. 138-144, 2019.

Wei Jiang (M'09-SM'19) received the Ph.D. degree in Computer Science from Beijing University of Posts and Telecommunications in 2008. From 2008 to 2012, he was with the 2012 Laboratory, HUAWEI Technologies. From 2012 to 2015, he was with Institute of Digital Signal Processing, University of Duisburg-Essen, Germany. Since 2015, he is a Senior Researcher with German Research Center for Artificial Intelligence (DFKI), which is the biggest European AI research institution and is the birthplace of "Industry 4.0" strategy. Meanwhile, he is a Senior Lecturer with University of Kaiserslautern, Germany. He is the author of three book chapters and over 60 conference and journal papers, holds around 30 granted patents, and participated in a number of EU and German research projects. He is an Associate Editor for IEEE Access and is a Moderator for IEEE TechRxiv.

Bin Han (M'15) received in 2009 his B.E. degree from Shanghai Jiao Tong University, M.Sc. in 2012 from Technical University of Darmstadt, and in 2016 the Ph.D. degree from Karlsruhe Institute of Technology. Since July 2016 he has been with University of Kaiserslautern as a Senior Lecturer, researching in the broad area of wireless communication and networking, with recent special focus on $\mathrm{B} 5 \mathrm{G} / 6 \mathrm{G}$ networks, network slicing, finite blocklength information theory, and information freshness. He is the author of over 30 conference and journal papers, and participated in multiple EU collaborative research projects. 
Mohammad Asif Habibi received his B.Sc. degree in Telecommunication Engineering from Kabul University, Afghanistan, in 2011. He obtained the M.Sc. degree in Systems Engineering and Informatics from Czech University of Life Sciences, The Czech Republic, in 2016. Since January 2017, he has been working as a Research Fellow and Ph.D. Candidate at Technische Universität Kaiserslautern, Germany. From 2011 to 2014, he joined HUAWEI, where he was working as a Radio Access Network Engineer. His main research interests include Network Slicing, Network Function Virtualization, Resource Allocation, Machine Learning, and Radio Access Network.

Hans D. Schotten (S'93-M'97) received the Ph.D. degrees from the RWTH
Aachen University of Technology, Germany, in 1997. From 1999 to 2003 , he worked for Ericsson. From 2003 to 2007, he worked for Qualcomm. He became manager of a R\&D group, Research Coordinator for Qualcomm Europe, and Director for Technical Standards. In 2007, he accepted the offer to become the full professor at the University of Kaiserslautern. In 2012, he - in addition - became scientific director of the German Research Center for Artificial Intelligence (DFKI) and head of the department for Intelligent Networks. Professor Schotten served as dean of the department of Electrical Engineering of the University of Kaiserslautern from 2013 until 2017. Since 2018, he is chairman of the German Society for Information Technology and member of the Supervisory Board of the VDE. He is the author of more than 200 papers and participated in 40+ European and national collaborative research projects. 